\title{
Role of Grafting in the Emulsion Polymerization of Vinyl Acetate with Poly(vinyl alcohol) as an Emulsifier. I. Effect of the Degree of Blockiness on the Kinetics and Mechanism of Grafting
}

\author{
B. M. BUDHLALl, * E. D. SUDOL, V. L. DIMONIE, A. KLEIN, M. S. El-AASSER \\ Emulsion Polymers Institute and Department of Chemical Engineering, Lehigh University, \\ 111 Research Drive, Bethlehem, Pennsylvania 18015
}

Received 24 April 2001; accepted 1 August 2001

\begin{abstract}
The role of grafting in particle nucleation during the emulsion polymerization of vinyl acetate with partially hydrolyzed poly(vinyl alcohol) (PVA) as an emulsifier and potassium persulfate as an initiator was investigated. The polymerizations were carried out in batch with a low solids (10\%) recipe. An automated reaction calorimeter (Mettler RC1) was used for the direct monitoring of the kinetics of emulsion polymerizations with three medium molecular weight PVAs differing in their degrees of blockiness (Poval 217EE $>217 \mathrm{E}>217$ ). Smith-Ewart case 1 kinetics (average number of free radicals per particle $<0.5$ ) were followed in all cases, and no constant rate in interval II was observed. Contrary to what was expected, a nonlinear relationship was observed between the rate of polymerization $\left(R_{\mathrm{p}}\right)$ and the number of particles $\left(N_{\mathrm{p}}\right)$. At $R_{\mathrm{p} \max } N_{\mathrm{p}}(217 \mathrm{E})>N_{\mathrm{p}}(217 \mathrm{EE})>N_{\mathrm{p}}$ (217), and the final $N_{\mathrm{p}}$ was independent of the degree of blockiness of PVA. The particle size distributions were broad (particle diameter $=20-100 \mathrm{~nm}$ ) and bimodal. On the basis of these data, we concluded that particle nucleation was continuous and was accompanied by extensive limited aggregation during the particle growth stages. The evolution of the amounts of grafted PVA and poly(vinyl acetate) (PVAc) were determined in polymerizations employing the two PVAs differing the most in blockiness (Poval 217EE and 217). The grafted PVAc followed similar profiles, increasing with conversion, particularly near the end of the two reactions. The amounts of grafted PVAc were about the same in the final latexes (37-39\%). In contrast, the grafting of PVA was nearly complete by the time monomer droplets had disappeared in each reaction (25\% conversion). However, the extent of grafting differed significantly, with the blockier PVA having about one-third the grafting of the more random PVA ( $\sim 10 \%$ vs $\sim 30 \%$ ). In these low solids recipes, grafting appeared to be primarily a solution event, occurring predominantly in the aqueous phase and not at the particle/water interface, as was previously speculated. The PVAc grafts grew until the molecules became water-insoluble and precipitated, forming polymer particles. (C $2001 \mathrm{John}$ Wiley \& Sons, Inc. J Polym Sci Part A: Polym Chem 39: 3633-3654, 2001
\end{abstract}

Keywords: poly(vinyl acetate); poly(vinyl alcohol); emulsion polymerization; reaction calorimetry; kinetics; grafting; grafting efficiency; particle size distribution

\section{INTRODUCTION}

Polymeric stabilization is typically exploited in the use of poly(vinyl alcohol) (PVA), sometimes

*Present address: Corporate Science and Technology Center, Air Products and Chemicals, Incorporated, Allentown, PA 18195

Correspondence to: M. S. El-Aasser (E-mail: mse0@lehigh.edu) Journal of Polymer Science: Part A: Polymer Chemistry, Vol. 39, 3633-3654 (2001) (c) 2001 John Wiley \& Sons, Inc. called a protective colloid, in the emulsion polymerization of vinyl acetate (VAc). The most effective polymeric stabilizers are considered to be amphipathic block or graft copolymers. The nominally insoluble part of the polymer anchors the soluble stabilizing moieties to the colloidal particles. When two particles collide, the mutually repulsive stabilizing moieties try to escape from the stress zone by either desorption from or lateral movement over the particle surface. Effective an- 
choring precludes both escape routes, and stability is imparted by osmotic and volume restriction repulsion. Medium molecular weight (77,000-79,000 $\mathrm{g} \mathrm{mol}^{-1}$ ), partially hydrolyzed ( $\left.\leq 90 \%\right)$, and blocky PVA stabilizes poly(vinyl acetate) (PVAc) latexes most effectively. ${ }^{1-3}$ The hydrophobic PVAc blocks (or grafts) provide the primary anchoring segments, and the hydrophilic PVA blocks project out into the aqueous phase as stabilizing moieties.

Extensive evidence supports grafting as a primary method of the attachment of PVA molecules to PVAc particles, ${ }^{4,5}$ but adsorption may also be significant. ${ }^{6}$ The PVAc blocks would comprise the trains, whereas the PVA blocks would form the tails and loops (chains terminally attached at one or both ends, respectively). These types of PVAs are uniquely suited to the emulsion polymerization of VAc because both the stabilizing anchors and latex particles are the same polymer, PVAc.

Commercial grades of PVA are customarily characterized by their acetyl content and the viscosity of their $4 \%$ aqueous solutions. Differences in the properties of PVA grades that meet the specification in acetyl content and $4 \%$ solution viscosity are caused by the possible structural variations in the polymer molecules, such as stereoregularity, intramolecular distribution of acetyl content, average acetyl length, broadness of molecular weight distribution, and frequency of long-chain and short-chain branching. ${ }^{7}$ The viscosity, particle size, stability, and freeze-thaw stability of the latex are determined largely by the molecular architecture of the particular PVA used in the polymerization.

Unable to arrive at an exact correlation to describe the influence of the acetyl content and molecular weight of PVA, Dunn ${ }^{4}$ explained his experimental results by the postulation of an uncontrolled factor that appears to be present in partially hydrolyzed PVA. According to this hypothesis, the polymerization may be retarded by the presence of conjugated unsaturated ketone groups produced by oxidation of the 1,2-glycol groups in the PVA molecule or, more likely, by the inhibiting action of a condensation product of acetaldehyde derived from hydrolysis of the residual VAc.

Some other authors do agree, however, on certain qualitative features of the reaction and role of PVA. ${ }^{2,3,5,8}$ Specifically, (1) hydrophobic acetate blocks aggregate, and hydrophilic blocks extend into the aqueous phase to form PVA pseudomicelles; (2) particles are generated continuously, primarily via a homogeneous nucleation mecha- nism; (3) VAc grafts onto partially hydrolyzed PVA chains, enhancing latex stability; and (4) conversion profiles are qualitatively similar to those obtained with ionic surfactants, but polymerization rate $\left(R_{\mathrm{p}}\right)$ values are typically lower.

Polymerization in the presence of polymeric surfactants alters the reaction kinetics and polymer structure caused by both transfer reactions to PVA and the specific character of the aqueous macromolecular aggregates. For ionically stabilized systems, empirical data and theoretical considerations support a homogeneous/coagulative particle nucleation mechanism with significant free-radical mobility (i.e., absorption into/desorption from latex particles) during most of the polymerization. A similar nucleation mechanism is assumed to prevail in PVA-stabilized systems because VAc remains relatively water-soluble. However, PVA pseudomicelles and grafting onto PVA chains suggest, at the least, altered kinetics. Because of the polymeric character of the stabilizers, the free-radical mobility may also be reduced, potentially altering every polymerization parameter, such as the average number of free radicals per particle $(\bar{n})$.

PVAs with different degrees of blockiness were shown to possess different hydrodynamic radii and cluster densities, leading to different conformations in the aqueous environment of an emulsion polymerization. ${ }^{9}$ These differences in the conformation may lead to differences in the rates of polymerization, the evolution of the number of particles $\left(N_{\mathrm{p}}\right)$, the amounts of grafted polymer, and the final latex properties. The question remains as to how this aggregation or micellization process affects the grafting reactions during the particle nucleation and particle growth stages.

This article focuses on further elucidating the role of grafting in particle nucleation during the emulsion polymerization of VAc with PVAs with different degrees of blockiness. Experiments were performed at a relatively low solids content (10\%) of PVAc. Based on the particle size distribution (PSD) and $N_{\mathrm{p}}$ for each sample analyzed, the total number of radicals per particle was estimated. With the acetonitrile $(\mathrm{AcN}) /$ hot-water selective solubilization technique developed by Magallanes González et al., ${ }^{10}$ the degree of grafting was measured. The determination of water-soluble, solvent-soluble, and solvent-insoluble species and their comparison with the initial composition give the amounts of grafted PVA (PVA-g-PVAc), branched PVAc (water- and solvent-insoluble), linear PVA (water-soluble), and linear PVAc (sol- 
vent-soluble). Selective solubilizations with AcN and water were used to separate the water-soluble PVA from the serum (aqueous phase) and particles and the AcN-soluble PVAc and waterinsoluble PVA-g-PVAc from the polymer phase.

\section{EXPERIMENTAL}

\section{Materials}

Three different PVA samples with different degrees of blockiness [Poval 217 (low), 217E (medium), and 217EE (high)] and similar degrees of polymerization ( $\mathrm{DP} \approx 1750$ ) were used in this study. These partially hydrolyzed PVAs (87-89\% hydrolyzed, as characterized by the supplier and confirmed by ${ }^{13} \mathrm{C}$ NMR) were all obtained as samples from Kuraray Co., Ltd. (Japan). For the emulsion polymerizations, VAc (Aldrich; >99\%) was distilled under nitrogen before each polymerization. Potassium persulfate (Sigma; 99.9\%) and sodium bicarbonate (BDH; 99.0\%) were used as supplied. Deionized water was used in all polymerizations. For the AcN/hot-water selective solubilization technique, AcN (Aldrich; >99.9\%, HPLC grade, $\mathrm{CH}_{3} \mathrm{CN}$, molecular weight $=41.05$, $\mathrm{bp}=81-82{ }^{\circ} \mathrm{C}$, density $=0.786$ ) was used as received for the extraction of PVAc homopolymer from the latex.

\section{AcN/Hot-Water Selective Solubilization Technique}

This method involves a two-step selective solubilization of latex components. In the first step, the linear VAc homopolymer is extracted with a solvent. AcN was chosen as the solvent because of its ability to dissolve PVAc selectively and to form an azeotrope with water at a relatively high concentration ( $16.3 \%$ water in the azeotrope ${ }^{11}$ ). In addition, the density of the solvent is relatively low; this facilitates the separation by ultracentrifugation of the AcN-insoluble components (i.e., PVA) from the PVAc-AcN solution. In the second step, the hot-water-soluble, mostly linear PVA is separated from the hot-water-insoluble, grafted PVA. In the entire extraction technique, the objective is to maximize the separation and minimize the loss.

\section{Rotary Evaporation}

The following solvent-exchange procedure was realized with a rotary evaporator (Buchi EL 131 Rotavapor). Approximately $10 \mathrm{~g}$ of latex was placed in a $120-\mathrm{mL}$ evaporating flask containing approximately $100 \mathrm{~mL}$ of AcN. The flask was then connected to the Rotavapor, where it was allowed to rotate at a constant rate of about $70 \mathrm{rpm}$ during the entire selective solubilization of PVAc in AcN. The temperature of the latex/AcN mixture was increased to $78{ }^{\circ} \mathrm{C}$ via a water bath, and the AcN/water azeotropic mixture was distilled at atmospheric pressure and condensed with a coldfinger condenser. The azeotrope started to boil at $76-78{ }^{\circ} \mathrm{C}$ under atmospheric pressure, and when the temperature of the final vapors reached $81-82{ }^{\circ} \mathrm{C}$ (boiling point of pure AcN), it was considered that all the water was removed from the mixture. The AcN-insoluble material (PVA) was separated via ultracentrifugation and rinsed two more times as described in the following section.

\section{Ultracentrifugation}

The separation of the AcN-insoluble material (PVA) was accomplished via ultracentrifugation of the AcN/PVAc/PVA mixture for $2 \mathrm{~h}$ at 37,000 rpm and $4{ }^{\circ} \mathrm{C}$. Polyallomer $10-\mathrm{mL}$ centrifugation tubes (polypropylene copolymer, Seton) were used. The insoluble materials (PVA) were sedimented at the bottom, whereas the supernatant containing the AcN-soluble PVAc was removed with a syringe. The centrifugation was repeated twice after redispersion of the sediment in fresh AcN. The AcN solutions collected after each centrifugation were added to the original one. The amount of PVAc solubilized was quantified by the drying of a known amount of PVAc/AcN solution and calculation of the total amount of PVAc based on the total amount of solution.

\section{Hot-Water Extraction}

An autoclavable bottle (120 mL, Teflon-lined cap) with its cap loosely engaged was used in the hotwater extraction of water-soluble PVA from the water-insoluble materials, primarily PVA-gPVAc. This bottle was placed on a hot plate/stirrer (VWR Scientific series 4000 HPS). The stirring speed was set at $400 \mathrm{rpm}$, and the temperature was set at $80{ }^{\circ} \mathrm{C}$ for $48 \mathrm{~h}$. This was done to separate the hot-water-soluble, mostly linear PVA from the hot-water-insoluble, grafted PVA. This extraction procedure was repeated on the separated solids after ultracentrifugation. The amount of PVA solubilized was then quantified by the drying of a known amount of PVA/water solution. The calculation of the water-soluble PVA was carried out on the basis of the total amount of 


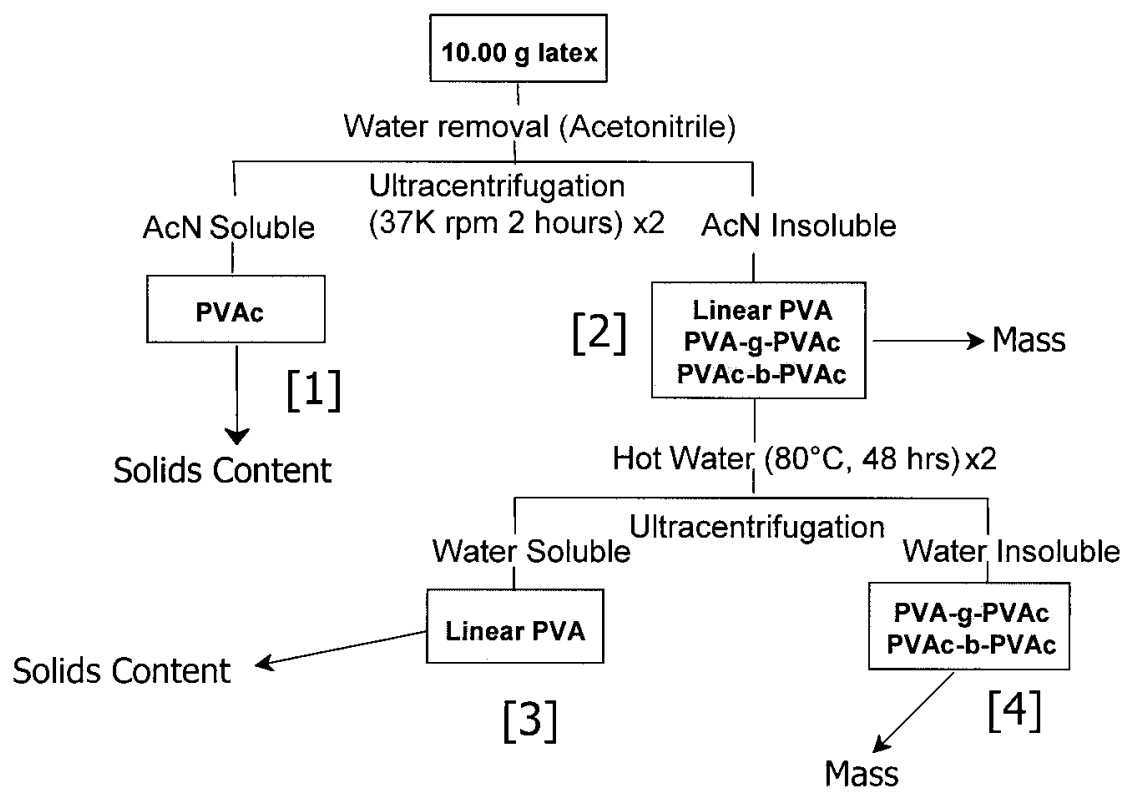

Figure 1. Polymer fractions obtained from the latex during the AcN/hot-water extraction process.

solution obtained. Repeatability of the double extraction method was good only after the complete extraction of PVAc homopolymer from the latex in the AcN-selective solubilization step. In the recipe for this study, the PVA/PVAc weight ratio was 0.425 in the final latex. As such, any remaining PVAc in the sample could cause an error in the quantification of the subsequent hot-water extraction by mass balance.

\section{Calculation of Grafting}

The extracted latex, as described previously, was divided into four polymer fractions, as shown in Figure 1:

1. The AcN-soluble PVAc.

2. The AcN-insoluble material containing linear PVA, grafted PVA, and branched PVAc.

3. The hot-water-soluble material, namely, linear PVA (water-soluble PVA), plus other water-soluble components, namely, potassium persulfate and sodium bicarbonate.

4. The hot-water-insoluble material (PVA) containing primarily grafted PVA but also possibly containing some branched PVAc that was insoluble in AcN (PVAc grafted, defined in eq 6 ).

The polymer fractions obtained by the extraction were then vacuum-dried and weighed. Quan- tification of the linear and grafted PVAc, branched PVAc, and linear and grafted PVA was performed by mass balance with the following:

PVA $\mathrm{w} / \mathrm{s}=$ Total $\mathrm{w} / \mathrm{s}$ fraction

$$
-\left(\mathrm{NaHCO}_{3}+\mathrm{KPS}\right)
$$

PVA grafted + PVAc grafted

+ PVAc branched $=$ Total w/ins fraction

$$
\text { PVA grafted }=\text { PVA recipe }-\mathrm{PVA} \text { w/s }
$$

PVA grafted $=$ PVA w/ins

$$
\text { - (VAc reacted - PVAc AcN/s) }
$$

PVAc grafted + PVAc branched

$$
=\mathrm{VAc} \text { reacted }-\mathrm{PVAc} \mathrm{AcN} / \mathrm{s}
$$

PVAc grafted + PVAc branched

$$
=\text { PVA } w / i n s-(\text { PVA recipe }- \text { PVA w/s })
$$

where AcN/s = AcN-soluble, w/s = water-soluble, and w/ins = water-insoluble. From the AcN solubilization step, the AcN-soluble PVAc fraction can be obtained, and from the hot-water extraction step, the total water-soluble and water-insoluble fractions can be obtained. With eqs $3-6$, the 
Table I. Recipe Used in the Batch Emulsion Polymerization of VAc with PVA at $60{ }^{\circ} \mathrm{C}$

\begin{tabular}{lc}
\hline \multicolumn{1}{c}{ Ingredient } & Weight $(\mathrm{g})$ \\
\hline Premix & \\
PVA (Poval 217, 217E, or & \\
217EE; 87-89\% hydrolyzed) & 32.0 \\
Deionized water & 768.0 \\
Emulsion & \\
Premix (4\% w/v PVA solution) & 614.0 \\
Deionized water & $111.4,^{\mathrm{b}} 0^{\mathrm{c}}$ \\
Sodium bicarbonate $\left(\mathrm{NaHCO}_{3}\right)$ & 0.15 \\
Potassium persulfate $\left(\mathrm{K}_{2} \mathrm{~S}_{2} \mathrm{O}_{8}\right)$ & $0.15\left(0.77 \mathrm{~m}^{\mathrm{a}}\right)$ \\
VAc & $58.3,{ }^{\mathrm{b}} 176^{\mathrm{c}}$ \\
\hline a Based on the aqueous phase. & \\
b Recipe based on 10\% solids. & \\
${ }^{\mathrm{c}}$ Recipe based on $25 \%$ solids.
\end{tabular}

amounts of PVA grafted and PVAc grafted (it is assumed that there is a negligible amount of PVAc branched material, and no further distinction is made in the calculations of the amount of PVAc grafted) can then be calculated from the experimental data. Equation 2 associates grafted PVA to grafted PVAc and is another method for calculating grafted PVA. Equations 3 and 4 represent two different methods of calculating PVA grafted, from water-soluble PVA and water-insoluble PVA, respectively. The total conversion percentage of VAc was determined by gravimetry. The grafting efficiency percentage of PVA and the grafting efficiency percentage of PVAc were calculated as defined by eqs 7 and 8 , respectively:

Grafting efficiency of PVA (\%)

$$
=\frac{\text { Grafted PVA }(\mathrm{g})}{\text { Total PVA }(\mathrm{g})} \times 100
$$

Grafting efficiency of PVAc (\%)

$$
\begin{gathered}
=\text { PVAc grafted }(\%) \\
=\frac{\text { Grafted monomer }(\text { VAc })(\mathrm{g})}{\text { Polymerized monomer }(\text { VAc })(\mathrm{g})} \times 100
\end{gathered}
$$

\section{Emulsion Polymerization Kinetics via Reaction Calorimetry}

The generic recipe used in the emulsion polymerization of VAc in the presence of the partially hydrolyzed PVAs with different degrees of blockiness is given in Table I. A kinetic and mechanistic investigation was performed on the emulsion po- lymerization of VAc with these PVAs and an investigation of the effect of a higher monomerwater ratio.

All reactions were batch emulsion polymerizations. These were carried out at $60^{\circ} \mathrm{C}$ in a Mettler $\mathrm{RC} 1$ reaction calorimeter equipped with a $1 \mathrm{~L}$ MP10 reactor with a baffle and turbine impeller. Calibration and ramping of the temperature to 60 ${ }^{\circ} \mathrm{C}$ were performed according to a procedure given by Varela de la Rosa. ${ }^{12}$ For each polymerization, aliquots (ca. $25 \mathrm{~mL}$ ) were removed by a syringe and needle at 5 -min intervals for the first $30 \mathrm{~min}$, then every $10 \mathrm{~min}$ for the next $30 \mathrm{~min}$, and then every 15 min thereafter until the end of the polymerization. To quench the reaction, each aliquot was transferred directly to a sample bottle that was placed in an ice/water bath and contained a preweighed quantity (ca. $1 \mathrm{~g}$ ) of a $1 \%$ aqueous hydroquinone solution. The samples were characterized in terms of PSDs and the amounts of grafted polymer to gain a better understanding of the role of grafting during the particle nucleation stage. Conversions were determined via both gravimetry and calorimetry. The heat of polymerization $\left(Q_{r}\right)$ was monitored to determine if there were characteristic reaction profiles for the different reaction conditions.

\section{Particle Size and PSD}

The evolution of the latex particle size and PSD was determined via capillary hydrodynamic fractionation (CHDF; Matec Applied Sciences model 1100). CHDF samples were sonified and filtered with a $5-\mu \mathrm{m}$ filter before injection into CHDF. The number-average $\left(D_{\mathrm{n}}\right)$, volume-average $\left(D_{\mathrm{v}}\right)$, and weight-average diameters $\left(D_{\mathrm{w}}\right)$ and PSD were obtained for each sample. Each sample was injected into $\mathrm{CHDF}$ at least twice, and the average values of $D_{\mathrm{n}}, D_{\mathrm{w}}$, and $D_{\mathrm{v}}$ are reported. $N_{\mathrm{p}}\left(\mathrm{dm}^{3}\right.$ of water $)$ was calculated from the gravimetric conversion and PSD with the following equation:

$$
N_{\mathrm{p}}=\frac{6 M_{\mathrm{o}} x_{\mathrm{g}}}{\pi \rho_{\mathrm{p}} \sum n_{i} D_{i}^{3}}
$$

where $M_{\mathrm{o}}$ is the initial amount of monomer added with respect to the water phase $\left(\mathrm{g} \mathrm{dm}^{-3}\right), x_{\mathrm{g}}$ is the fractional gravimetric conversion for each sample obtained, $\rho_{\mathrm{p}}$ is the polymer density $\left(1191 \mathrm{~g} \mathrm{dm}^{-3}\right.$ for PVAc), and $n_{i}$ is the fraction of particles with diameter $D_{i}(\mathrm{~m})$. 


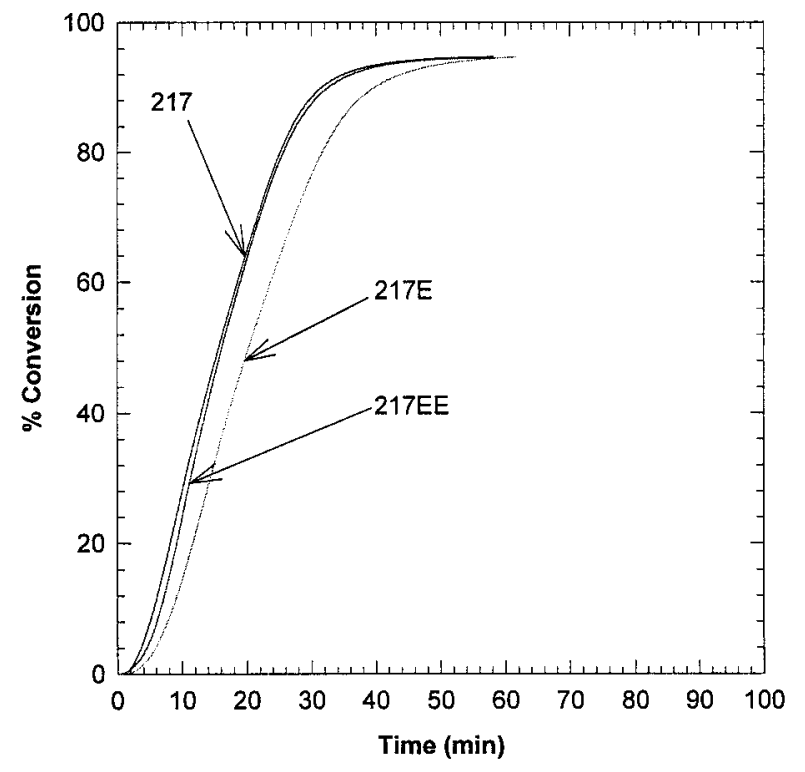

Figure 2. Evolution of the calorimetric conversiontime curves for the emulsion polymerization of VAc with PVAs with different degrees of blockiness: Poval 217 (low), Poval 217E (medium), and Poval 217EE (high); $\left(\mathrm{K}_{2} \mathrm{~S}_{2} \mathrm{O}_{8}=0.77 \mathrm{mM}, T_{\mathrm{r}}=60{ }^{\circ} \mathrm{C}, 10 \%\right.$ solids).

\section{RESULTS AND DISCUSSION}

\section{Effect of the Degree of Blockiness of PVA}

\section{Kinetic Studies}

In an attempt to further understand the role of grafting reactions in the mechanism of particle nucleation in the emulsion polymerization of VAc with PVA as an emulsifier, we decided to further investigate the effect of the different degrees of blockiness of the PVAs on the emulsion polymerization kinetics. Figure 2 illustrates the conversion-time behaviors for VAc polymerizations carried out at 60 ${ }^{\circ} \mathrm{C}$ with the three PVAs (recipe in Table I) and 0.77 $\mathrm{mM}$ potassium persulfate $\left(\mathrm{K}_{2} \mathrm{~S}_{2} \mathrm{O}_{8}\right)$. For VAc homopolymerization, the calorimetric and gravimetric conversions are coincident, and only the calorimetric curves are reported here.

Typically, the experimental conversion-time curves exhibit a short induction period followed by a rapid increase in the rate. The curves then appear to become nearly linear, which could be interpreted as a constant rate period, but, as we will see from the $Q_{\mathrm{r}}\left(R_{\mathrm{p}}\right)$ data, no true constant rate period is observed.

As seen in Figure 2, the PVA with the lowest degree of blockiness (Poval 217) gave the highest initial and overall $R_{\mathrm{p}}$ 's. The polymeric-stabilized latex system is reasonably shear-stable, and the degree of agitation used in this experiment (400 $\mathrm{rpm}$ ) should be adequate to maintain reproducible kinetics. The next fastest was that performed with the PVA with the highest degree of blockiness (217EE). From reproducibility experiments, it was found that the polymerization kinetics of Poval 217EE and 217 were interchangeable, and these are considered to be within experimental error of each other. The third PVA (217E), with a medium degree of blockiness, led to slower $R_{\mathrm{p}}$ values.

Figures 3 and 4 show the evolution of $Q_{\mathrm{r}}$ and $R_{\mathrm{p}}$ as a function of time and conversion, respectively, for the three PVAs studied. All $Q_{\mathrm{r}}$ data were recorded every $6 \mathrm{~s}$. The calorimetric curves in Figure 2 were obtained by the integration of $Q_{r}$-time curves.

When $R_{\mathrm{p}}$ is plotted against both time and conversion, similarities and differences can be distinguished in the kinetics. First note that the maximum in the rate $\left(R_{\mathrm{p} \max }\right)$ was obtained at about the same conversion for all the experiments (26-28\% conversion). This maximum does not indicate the end of nucleation, as for the emulsion polymerization of styrene, ${ }^{13}$ because $N_{\mathrm{p}}$ continued to increase past this point, as discussed in the next section. This is, however, in the range of conversions reported for the disappearance of VAc monomer droplets and is taken here as evidence for this phenomenon.

Another characteristic observed in the $R_{\mathrm{p}}$ curves for all the experiments is a plateau or shoulder between about 50 and $75 \%$ conversion. This is considered to be the result of the Trommsdorf or gel effect occurring under these isothermal conditions. Although more commonly seen with such monomers as styrene and methyl methacrylate, some researchers have also observed this feature in VAc polymerizations. ${ }^{14}$ In this case, however, this autoacceleration effect occurs at an earlier stage in the reaction than reported in the literature (normally ca. $85 \%$ conversion). This raises questions concerning its origin and whether or not this feature is indeed caused by what is normally characterized as the gel effect or an anomaly of low percent solid polymerizations. Under these low solids conditions, the VAc partitions substantially between the aqueous and particle phases at these conversions, decreasing the monomer concentration in the particles $\left([\mathrm{M}]_{\mathrm{p}}\right)$, resulting in an increase in $\bar{n}$, thus increasing $R_{\mathrm{p}}$. The possible reasons for this feature are discussed later.

$N_{p}$

With the goal of gaining a better understanding of this reaction, we followed the evolution of $N_{\mathrm{p}}$ 


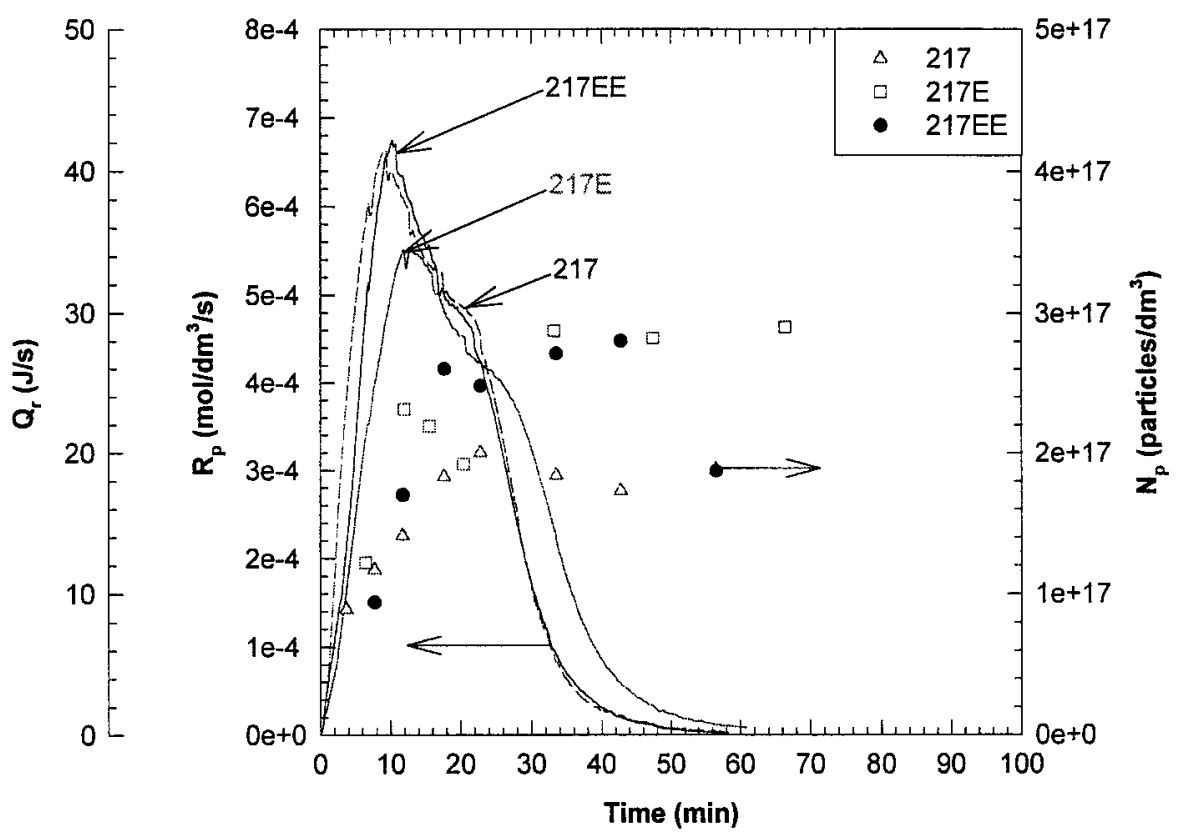

Figure 3. $R_{\mathrm{p}}$ and the evolution of $N_{\mathrm{p}}$ versus time for the emulsion polymerization of VAc with PVAs with different degrees of blockiness: Poval 217 (low), Poval 217E (medium), and Poval 217EE (high); $\left(\mathrm{K}_{2} \mathrm{~S}_{2} \mathrm{O}_{8}=0.77 \mathrm{mM}, T_{\mathrm{r}}=60{ }^{\circ} \mathrm{C}, 10 \%\right.$ solids).

(PSDs measured by CHDF), and we present it as a function of time and conversion in Figures 3 and 4 , respectively. For all PVAs, the evolution of $N_{\mathrm{p}}$ indicated the existence of both continuous nucle- ation and limited aggregation (note that no measurable coagulum was found in these latexes). This is indicated by the increase to maxima, followed by decreases, increases, and so forth in the

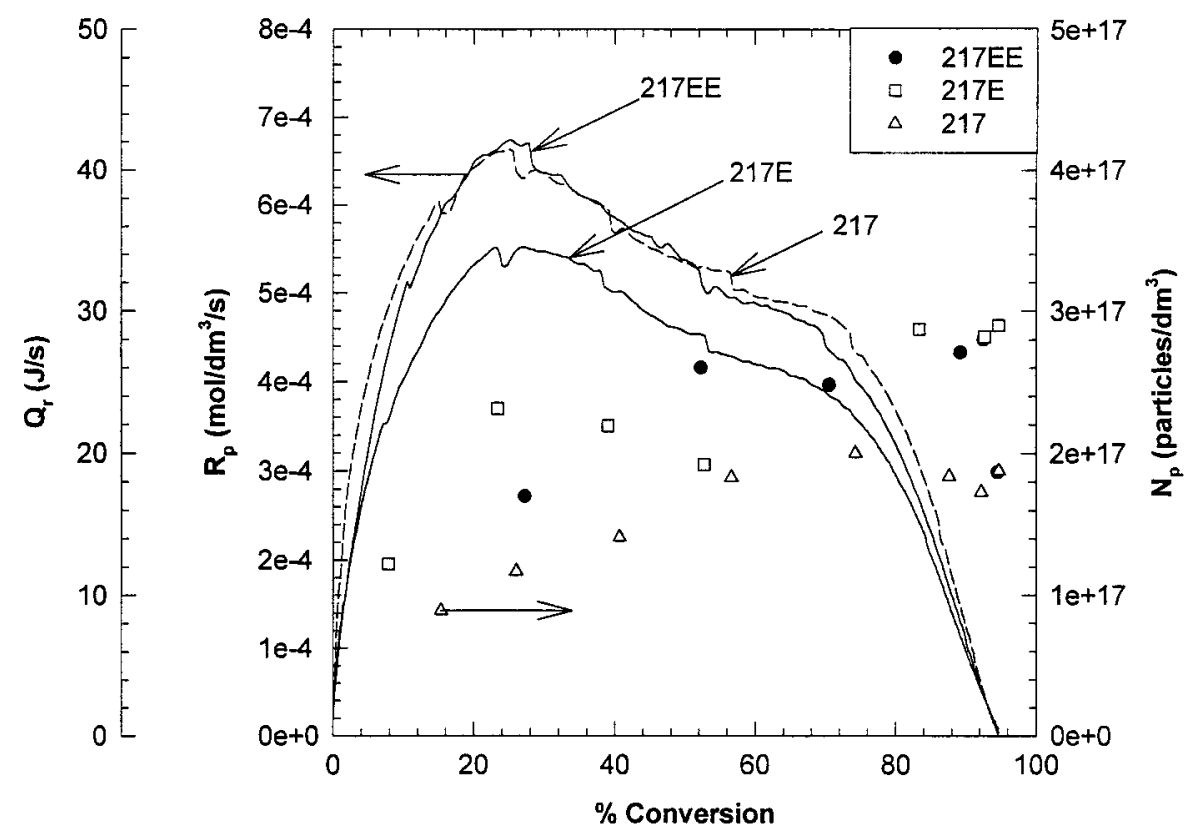

Figure 4. $R_{\mathrm{p}}$ and the evolution of $N_{\mathrm{p}}$ versus conversion for the emulsion polymerization of VAc with PVAs with different degrees of blockiness: Poval 217 (low), Poval 217E (medium), and Poval 217EE (high); $\left(\mathrm{K}_{2} \mathrm{~S}_{2} \mathrm{O}_{8}=0.77 \mathrm{mM}, T_{\mathrm{r}}=60{ }^{\circ} \mathrm{C}, 10 \%\right.$ solids). 


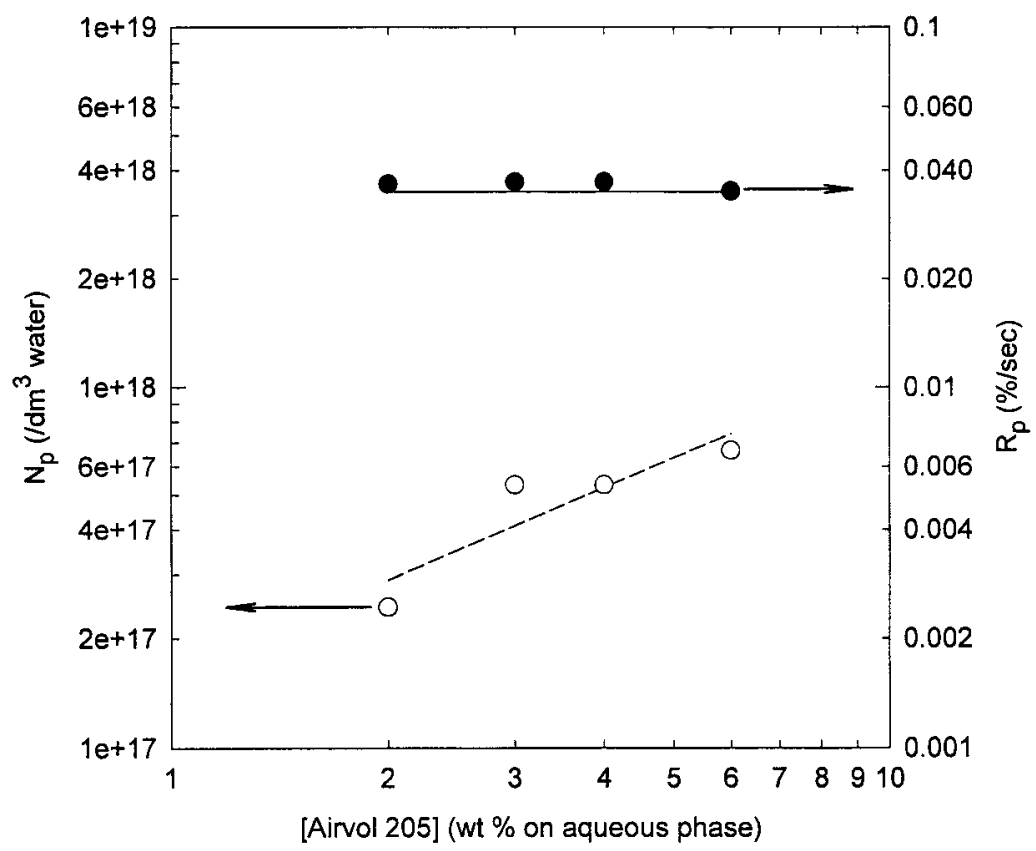

Figure 5. Effect of the Airvol 205 concentration on $N_{\mathrm{p}}$ and $R_{\mathrm{p}}$ (Airvol $=3 \%, \mathrm{~K}_{2} \mathrm{~S}_{2} \mathrm{O}_{8}$ $=10 \mathrm{mM}, T_{\mathrm{r}}=50{ }^{\circ} \mathrm{C}, 30 \%$ solids; experimental data taken from ref. 2 ).

particle numbers. These results show no obvious relationship between $R_{\mathrm{p}}$ and $N_{\mathrm{p}}$. At $R_{\mathrm{p} \max }, N_{\mathrm{p}}$ $(217 \mathrm{E})>N_{\mathrm{p}}(217 \mathrm{EE})>N_{\mathrm{p}}(217)$. It was observed from reproducibility experiments that $R_{\mathrm{p}}$ was apparently independent of the measured $N_{\mathrm{p}}$ (i.e., $R_{\mathrm{p}}$ $\propto N_{\mathrm{p}}{ }^{x}$, where $x=0$ ). It was postulated previous$1 y^{15}$ that the PVA with the lowest degree of blockiness would have a random coil conformation in the aqueous phase that would result in a decreased ability to stabilize polymer particles, leading to a lower $N_{\mathrm{p}}$ compared with that of the blockier PVAs, which would have a pseudomicellar type of structure in the aqueous phase and possess better emulsifier properties. This would qualitatively agree with the traditional behavior seen in emulsion polymerization, where a greater $N_{\mathrm{p}}$ is usually obtained for a better emulsifier.

Pramojaney ${ }^{2}$ also observed an independence of $R_{\mathrm{p}}$ on $N_{\mathrm{p}}$ and the emulsifier concentration for the emulsion polymerization of VAc with PVA (Airvol 205, 88\% partially hydrolyzed, molecular weight $=35,000 \mathrm{~g} / \mathrm{mol}$; Air Products \& Chemicals, Inc.). Figure 5 is a plot of the reported results showing the influence of the PVA (Airvol 205, 30\% solids latex) concentration on $R_{\mathrm{p}}(\% / \mathrm{s})$ and $N_{\mathrm{p}}\left(\mathrm{mol} / \mathrm{cm}^{3}\right)$, as obtained by Pramojaney. ${ }^{2}$ The empirical relationships are given as $R_{\mathrm{p}} \propto[\mathrm{Airvol} 205]^{0}$ and $N_{\mathrm{p}} \propto$ [Airvol] ${ }^{0.9}$. There were no conclusive explanations for these relationships, except that the technique used to measure the PSD (electron microscopy) may underestimate the presence of very small particles. These particles were often less than 30 $\mathrm{nm}$ and difficult to measure from the micrographs because they tended to aggregate or deposit on larger particles, thus making themselves invisible.

In this study, two reasons for the inconsistent relationship observed between $R_{\mathrm{p}}$ and $N_{\mathrm{p}}$ for Poval 217EE, 217E, and 217 were considered. First, some of the latex samples may not have been sufficiently stable to withstand the sampling and storage conditions before the particle size measurements. Thus, a lower $N_{\mathrm{p}}$ would result from the measurement than was actually present in the reactor at the time of sampling. Second, $\bar{n}$ becomes high, which causes the rate to increase, even though $N_{\mathrm{p}}$ is relatively low. An attempt to estimate and compare values of $\bar{n}$ is given in the following section.

\section{Calculation of $[\mathrm{M}]_{p}$ during Interval III}

To examine the second possible cause, $\bar{n}$ was calculated with respect to conversion for the three polymerizations. The values of $N_{\mathrm{p}}$ were taken from the results given in Figure 4, and the propagation coefficient $\left(k_{\mathrm{p}} ; \mathrm{dm}^{3} / \mathrm{mol} \mathrm{s}\right)$ at the polymerization temperature $(T=333 \mathrm{~K})$ was calculated with the Arrhenius relationship ${ }^{16}$ given by 


$$
\ln k_{p}=(19.4 \pm 0.7)-(3350 \pm 200) / T
$$

Because monomer droplets are usually thought to act solely as reservoirs of monomer (essentially because their total surface area is much less than that of particles) and because the latex particles imbibe monomer as polymer is produced within them, eventually the monomer droplets become exhausted (26-28\% conversion), at which point the droplet phase disappears. To this point, $[\mathrm{M}]_{\mathrm{p}}$ is typically considered to be constant (except for very small particles). However, after this stage the monomer will only be in the latex particles and the aqueous phase, as governed by the thermodynamic equilibrium; and so polymerization continues to consume the remaining monomer. After this point, $[\mathrm{M}]_{\mathrm{p}}$ is a function of conversion. Integrating to obtain $x$, the fractional conversion, from calorimetry, we can calculate $[\mathrm{M}]_{\mathrm{p}}$ by carrying out a mass balance on the monomer, as given by eq 11:

$$
M=M_{0}-M_{0} x=W_{\mathrm{VAc} / \mathrm{PVAc}} x M_{0}+M_{\mathrm{aq}}+M_{\mathrm{droplets}}
$$

where $M_{0}$ is the initial monomer/water ratio ( $\mathrm{g}$ $\left.\mathrm{dm}^{-3}\right) ; W_{\mathrm{VAc} / \mathrm{PVAc}}$ is the monomer/polymer weight ratio in the polymer particles; and $M_{\text {aq }}$ and $M_{\text {droplets }}$ are the amounts of monomer dissolved in the water phase and present in the droplets ( $\mathrm{g}$ $\mathrm{dm}^{-3}$ ), respectively. Thus, once the monomer droplets disappear, at $x$, where $R_{\mathrm{p}}$ is maximum, and with a value for the saturation concentration of VAc monomer in the aqueous phase ${ }^{2}$ of 2.858 $\times 10^{-2} \mathrm{~g} / \mathrm{cm}^{3}$, eq 11 becomes

$$
W_{\mathrm{VAc} / \mathrm{PVAc}}=\left[\left(1-2.858 \times 10^{-2} / M_{0}\right)-x\right] / x
$$

The conversion at which the monomer droplets disappear is considered to occur at the maximum in $R_{\mathrm{p}}\left(R_{\mathrm{p} \text { max }}\right)$ because after this point $R_{\mathrm{p}}$ decreases because of the decrease of the monomer concentration in the particles (and, as such, no constant rate occurs during interval III). Figure 3 is used to obtain this conversion. The monomer concentration in the polymer particles is then calculated with eq 13 :

$$
[\mathrm{M}]_{\mathrm{p}}=\frac{W_{\mathrm{VAc} / \mathrm{PVAc}} \rho_{\mathrm{m}} \rho_{\mathrm{p}} \times 10^{3}}{\left(W_{\mathrm{VAc} / \mathrm{PVAc}} \rho_{\mathrm{p}}+\rho_{\mathrm{m}}\right) \mathrm{MW}}
$$

where $\rho_{\mathrm{m}}\left(0.934 \mathrm{~g} / \mathrm{cm}^{3}\right)$ and $\rho_{\mathrm{p}}\left(1.191 \mathrm{~g} / \mathrm{cm}^{3}\right)$ are the monomer and polymer densities, respectively, and MW is the molecular weight of VAc (86 $\mathrm{g} / \mathrm{mol})$. $[\mathrm{M}]_{\mathrm{p}}$ is calculated as a function of conver-

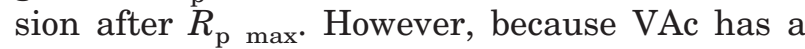
high water solubility, the partitioning of the monomer into the aqueous phase must be taken into account. To determine this parameter, we derived five equations, and we developed and transformed a general quadratic expression to obtain the unknowns: the mass of monomer in the particles $\left(G_{\mathrm{m} / \mathrm{p}}\right)$, the mass of monomer in the aqueous phase $\left(G_{\mathrm{m} / \mathrm{w}}\right)$, the concentration of monomer in the particles $\left([\mathrm{M}]_{\mathrm{p}}\right)$, the concentration of monomer in the aqueous phase $\left([\mathrm{M}]_{\mathrm{w}}\right.$,), the volume of monomer in the particles $\left(V_{\mathrm{p}}\right)$, and the volume of monomer in the aqueous phase $\left(V_{\mathrm{aq}}\right)$. If it is assumed that there is no barrier to a monomer molecule or radical diffusing from the water into a particle, then the positive change in interaction energy on diffusion from a particle into water will increase the time a radical is held within the particle by the ratio of the solubilities of the monomer in the two phases. This is the minimum increase in time because it is assumed that there is no interfacial barrier, as the chain transferred vinyl radical is very similar to monomer. It is assumed that the equilibrium partition coefficient $k_{\mathrm{a}}$ (where $k_{\mathrm{a}}=[\mathrm{M}]_{\mathrm{p}} /[\mathrm{M}]_{\mathrm{w}}$ ) is constant up to the disappearance of droplets. That is, the rate of monomer consumption within the particle is the same as the rate of monomer diffusion from the aqueous phase. These unknown parameters are expressed quantitatively in eqs 14-19:

$$
k_{\mathrm{a}}=\frac{[\mathrm{M}]_{\mathrm{p}}}{[\mathrm{M}]_{\mathrm{w}}}=20.1=\frac{\left[\frac{G_{\mathrm{m} / \mathrm{p}}}{\mathrm{MW}} \times \frac{1}{V_{\mathrm{p}}}\right]}{\left[\frac{G_{\mathrm{m} / \mathrm{w}}}{\mathrm{MW}} \times \frac{1}{V_{\mathrm{aq}}}\right]}
$$

At $60{ }^{\circ} \mathrm{C},[\mathrm{M}]_{\mathrm{p}}$ at $R_{\mathrm{p} \text { max }}$ has been found in different studies to range from 6.3 to $7.6 \mathrm{~mol} / \mathrm{dm}^{3}$ in the polymer particles. ${ }^{17}$ In this study, an average value for $[\mathrm{M}]_{\mathrm{p}}$ of $6.9 \mathrm{~mol} / \mathrm{dm}^{3}$ was found:

$$
\begin{gathered}
G_{\mathrm{m} / \mathrm{p}}+G_{\mathrm{m} / \mathrm{w}}=G_{\mathrm{m}}(x)=(1-x) G_{\mathrm{m}}(0) \\
V_{\mathrm{p}}=\frac{G_{\mathrm{m} / \mathrm{p}}}{\rho_{\mathrm{m}} \times 1000}+\frac{G_{\mathrm{p} / \mathrm{p}}}{\rho_{\mathrm{p}} \times 1000} \\
V_{\mathrm{aq}}=\frac{G_{\mathrm{m} / \mathrm{p}}}{\rho_{\mathrm{m}} \times 1000}+\frac{G_{\mathrm{p} / \mathrm{p}}}{\rho_{\mathrm{p}} \times 1000}
\end{gathered}
$$




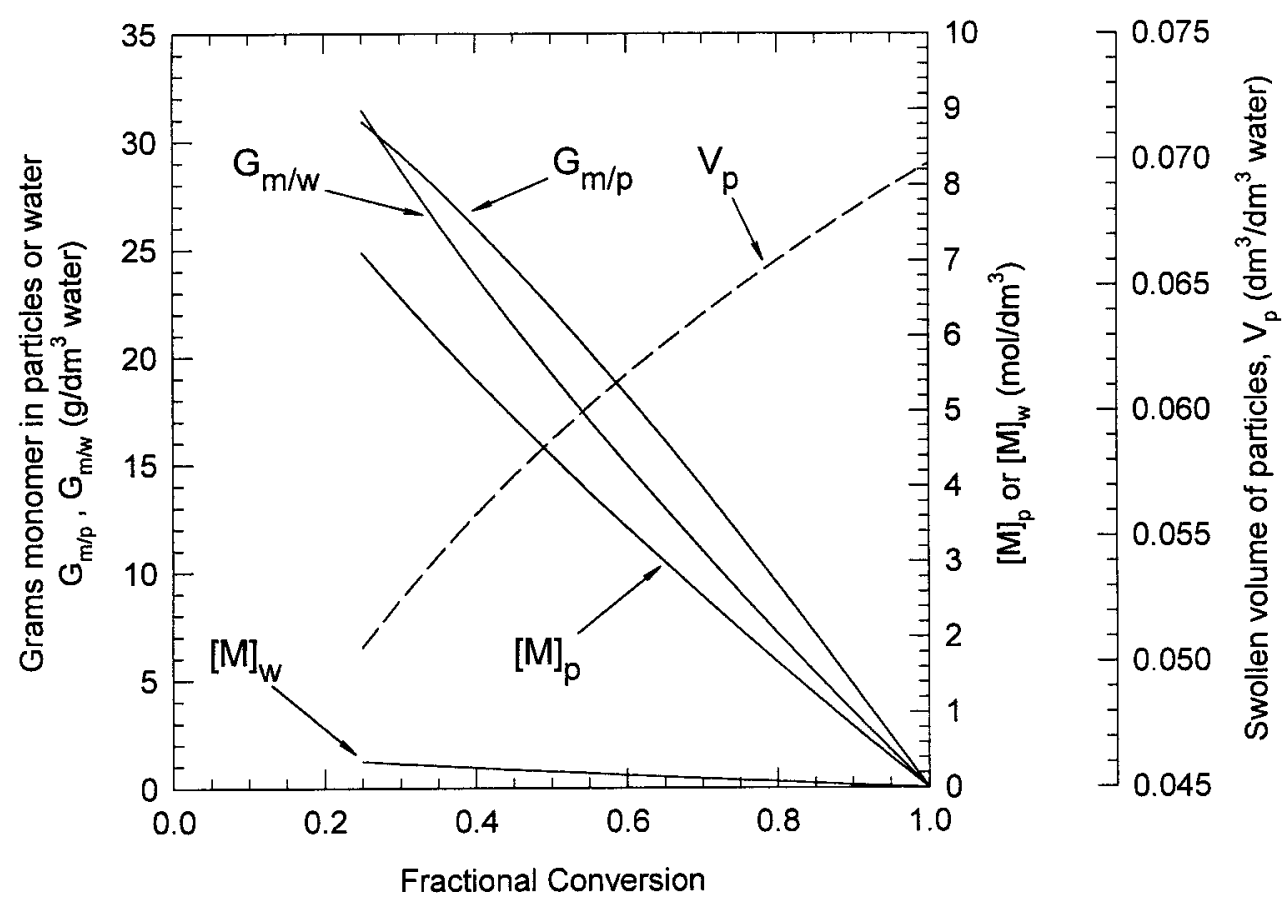

Figure 6. Evolution of the monomer concentration (monomer/water ratio, $M_{\mathrm{o}}=83$ $\left.\mathrm{g} / \mathrm{dm}^{3}\right)$ in the particles $\left([\mathrm{M}]_{\mathrm{p}}\right)$ and aqueous phase $\left([\mathrm{M}]_{\mathrm{w}}\right)$ and the corresponding evolution of the total swollen volume of the particles $\left(V_{\mathrm{p}}\right)$. The grams of monomer present in both phases $\left(G_{\mathrm{m} / \mathrm{w}}\right.$ and $\left.G_{\mathrm{m} / \mathrm{p}}\right)$ are also represented.

$$
\begin{gathered}
G_{\mathrm{p} / \mathrm{p}}=x \times G_{\mathrm{m}}(0) \\
\frac{(k-1) G_{\mathrm{m} / \mathrm{p}}^{2}}{\rho_{\mathrm{m}} \times 1000}+G_{\mathrm{m} / \mathrm{p}}\left[\frac{(1-x) G_{\mathrm{m}}(0)}{\rho_{\mathrm{m}} \times 1000}+\frac{G_{\mathrm{w}}}{\rho_{\mathrm{w}} \times 1000}\right. \\
\left.-\frac{k(1-x) G_{\mathrm{m}}(0)}{\rho_{\mathrm{m}} \times 1000}+\frac{k x\left[G_{\mathrm{m}}(0)\right]}{\rho_{\mathrm{p}} \times 1000}\right] \\
-\frac{k x(1-x) G_{\mathrm{m}}(0)^{2}}{\rho_{\mathrm{p}} \times 1000}=0
\end{gathered}
$$

Equation 19 (a quadratic equation) is solved for $G_{\mathrm{m} / \mathrm{p}}$ for a given value of $x$. From this, $G_{\mathrm{p} / \mathrm{p}}, V_{\mathrm{aq}}$, $V_{\mathrm{p}},[\mathrm{M}]_{\mathrm{p}}$, and $[\mathrm{M}]_{\mathrm{w}}$ are calculated. Figure 6 illustrates the variation in $[\mathrm{M}]_{\mathrm{p}},[\mathrm{M}]_{\mathrm{w}}, G_{\mathrm{m} / \mathrm{p}}, G_{\mathrm{m} / \mathrm{w}}$, and $V_{\mathrm{p}}$ with conversion for the system containing initially $8.3 \mathrm{wt} \%$ VAc based on the water $(10 \%$ solid recipe). The values of $[\mathrm{M}]_{\mathrm{p}}$ can now be used to calculate the values of $\bar{n}$. Note that although the monomer concentrations in the water $\left([\mathrm{M}]_{\mathrm{w}}\right)$ and particles $\left([\mathrm{M}]_{\mathrm{p}}\right)$ differ substantially, the low phase ratio of particles to water results in similar amounts of monomer in each of these phases, $G_{\mathrm{m} / \mathrm{w}}$ and $G_{\mathrm{m} / \mathrm{p}}$, respectively. One consequence of this is that in interval III, the total volume of the particles $\left(V_{\mathrm{p}}\right)$ actually increases by $40 \%$ from the moment that droplets disappear to full conversion. This is because monomer in the particles is continuously replenished by monomer diffusing from the aqueous phase, a large source of monomer in this case. This is the opposite of what normally happens in interval III of a typical emulsion polymerization where the swollen volume decreases with increasing conversion. Indeed, this is the case for less water-soluble monomers and even for VAc at a more typical solids content (e.g., 30-50\%). This increase in the volume of each particle may be another factor contributing to an increase in $\bar{n}$, thus leading to an increase in $R_{\mathrm{p}}$ previously attributed to the gel effect.

The estimated values of $\bar{n}$ at $R_{\mathrm{p} \text { max }}$ for the experiments performed with the three PVAs with different degrees of blockiness are shown in Table II, and their evolution with conversion is illustrated in Figure 7 (curves drawn between points have been added only to aid in following the data). First note that all values of $\bar{n}$ are well below 0.5 throughout all these reactions, fitting case $1 \mathrm{ki}-$ netics of the Smith-Ewart theory. ${ }^{18}$ This reflects the importance of the radical-transfer/desorption process in the latex particle with termination in 
Table II. Kinetic Details Obtained for VAc Emulsion Polymerizations with PVAs with Different Degrees of Blockiness $^{\mathrm{a}}$

\begin{tabular}{|c|c|c|c|c|c|c|}
\hline PVA & $\begin{array}{c}R_{\mathrm{p} \max } \times 10^{-4} \\
\left(\mathrm{~mol} / \mathrm{dm}^{3} / \mathrm{s}\right)\end{array}$ & $\begin{array}{c}x @ R_{\mathrm{p} \max } \\
(\%)\end{array}$ & $\begin{array}{c}\text { Final } D_{\mathrm{v}} \\
\text { at } R_{\mathrm{p} \max } \\
(\mathrm{nm} ; \mathrm{CHDF})\end{array}$ & $\begin{array}{c}\text { Final } \\
N_{\mathrm{p}} \times 10^{-17} \\
\text { at } R_{\mathrm{p} \max } \\
\left(\text { particles } / \mathrm{dm}^{3}\right)\end{array}$ & $\begin{array}{c}{[\mathrm{M}]_{\mathrm{p}} \text { at }} \\
R_{\mathrm{p} \max } \\
\left(\mathrm{mol} / \mathrm{dm}^{3}\right)\end{array}$ & $\begin{array}{c}\bar{n} \text { at } \\
R_{\mathrm{p} \text { max }}\end{array}$ \\
\hline Poval 217 & 6.6 & 25 & 66.4 & 1.14 & 6.9 & 0.041 \\
\hline Poval 217E & 5.5 & 27 & 50.9 & 2.73 & 7.0 & 0.018 \\
\hline Poval 217EE & 6.8 & 25 & 59.3 & 1.60 & 6.9 & 0.031 \\
\hline
\end{tabular}

${ }^{\mathrm{a}} \mathrm{K}_{2} \mathrm{~S}_{2} \mathrm{O}_{8}=0.77 \mathrm{mM}, T_{\mathrm{r}}=60{ }^{\circ} \mathrm{C}, 10 \%$ solids.

the aqueous phase. These values are in accordance with those reported in the literature $(\bar{n}$ $\sim 0.1$ or lower for a variety of reaction conditions). ${ }^{19}$ The trend observed for all three reactions at high conversions $(>50 \%)$ is an increasing value of $\bar{n}$ up to a maximum, followed by a decrease at the end of the polymerization.

In a comparison of the kinetics obtained for Poval 217 and $217 \mathrm{EE}$, the similar $R_{\mathrm{p}}$ 's cannot be explained by the results obtained for $N_{\mathrm{p}} . \bar{n}$ is higher for Poval 217 than for 217EE. This is at least partially a consequence of the larger particle size (lower $N_{\mathrm{p}}$ ), which leads to less desorption of radicals. Therefore, desorption and termination in the aqueous phase seem to be relatively more important for Poval 217EE PVA.

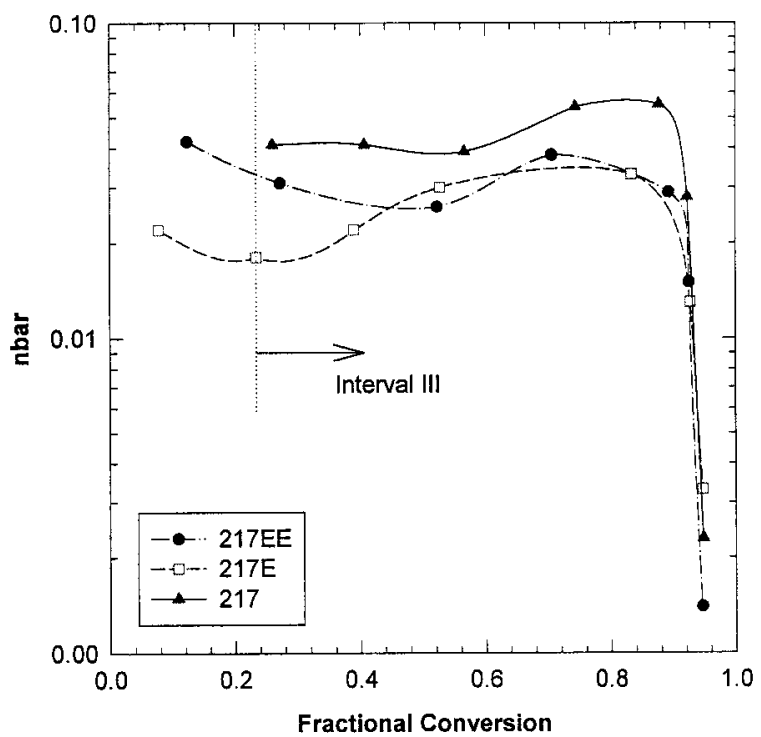

Figure 7. $\bar{n}$ versus conversion as a function of the degree of blockiness of PVA for the batch emulsion polymerization of VAc $\left(\mathrm{K}_{2} \mathrm{~S}_{2} \mathrm{O}_{8}=0.77 \mathrm{mM}, T_{\mathrm{r}}=60{ }^{\circ} \mathrm{C}\right.$, $10 \%$ solids).
It is known that the rate at which the monomer enters a particle is diffusion-controlled. Whether or not this rate of monomer diffusion from the aqueous phase into the polymer particles will be influenced by the degree of blockiness of the PVA used (i.e., the extent of its adsorption onto the polymer particles) has yet to be determined. In addition, whether or not the degree of blockiness or the extent of branching and grafting of the PVA influences the entry and exit rate of the radicals into the particles (as may be indicated by the kinetics) has yet to be understood and cannot be dismissed as a possible contributor to the observed kinetics.

\section{PSD Analysis}

To gain further understanding of the particle nucleation mechanisms using the three PVAs, the evolution of the PSDs were monitored by CHDF. The number distributions were first normalized to the amount of polymer produced (based on the fractional conversion). This was done by the adjustment of the area under each of the number fraction- $D^{3}$ ( $\propto$ particle volume) curves to be proportional to the amount of polymer produced. A new number distribution curve (relative number of particles vs $D$ ) was obtained normalized to the weight of polymer (reference distribution arbitrarily chosen as the lowest conversion sample of Poval 217). These are illustrated in Figure 8(a-c). These plots allow a relative comparison of the evolution of the PSD for each reaction.

A comparison of the final (95\% conversion) samples for Poval 217, 217E, and 217EE is shown in Figure 9. The final PSDs for all the polymerizations were found to be broad (particle diameter $=20-100 \mathrm{~nm}$ ) and bimodal. For all polymerizations, there appears to be a continuous generation of new particles (continuous nucleation), as evi- 


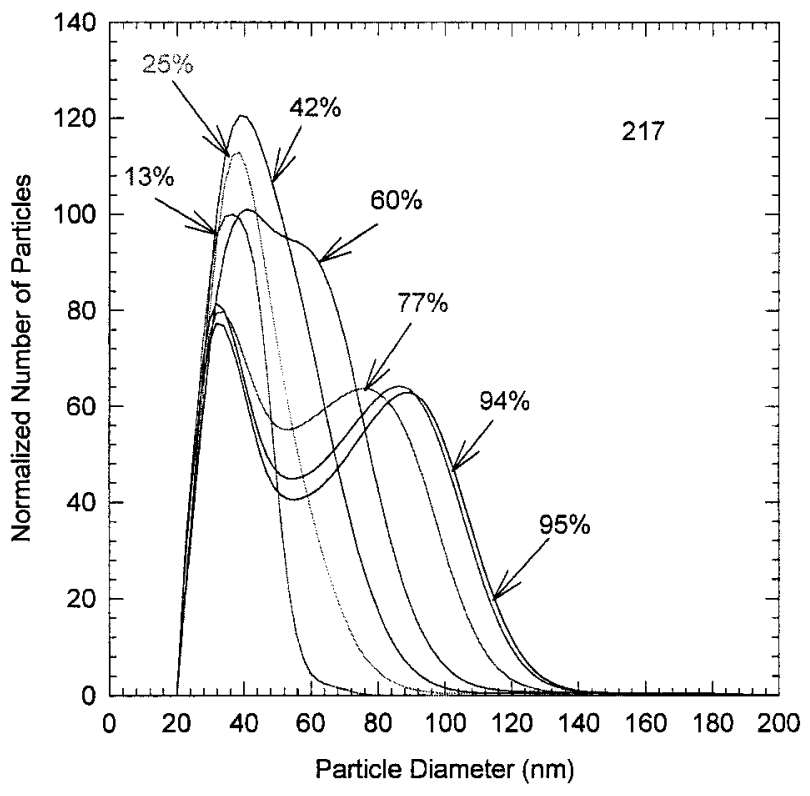

(a)

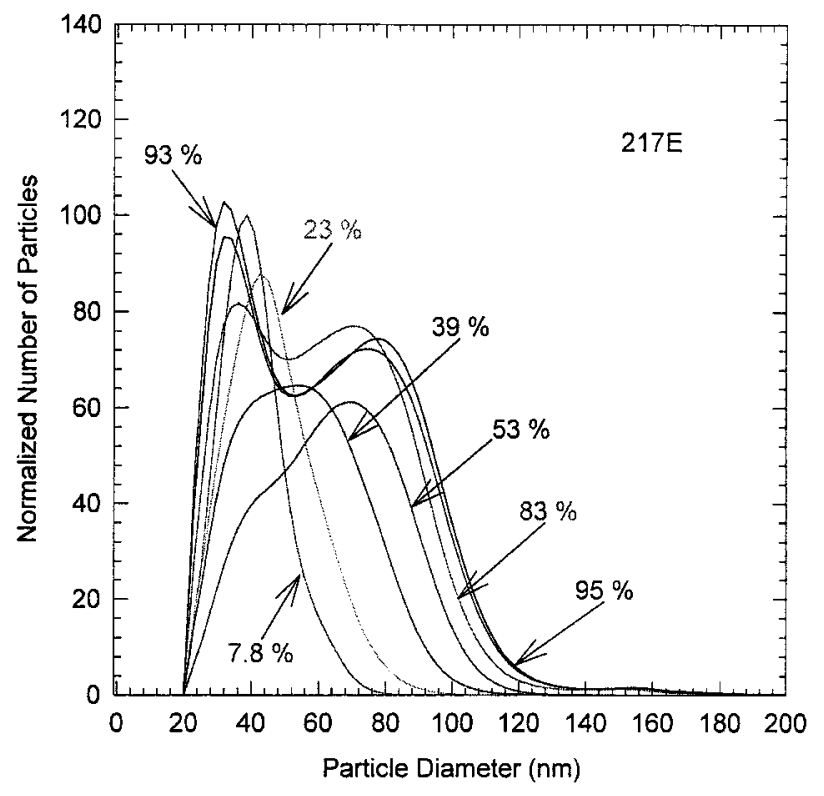

(b)

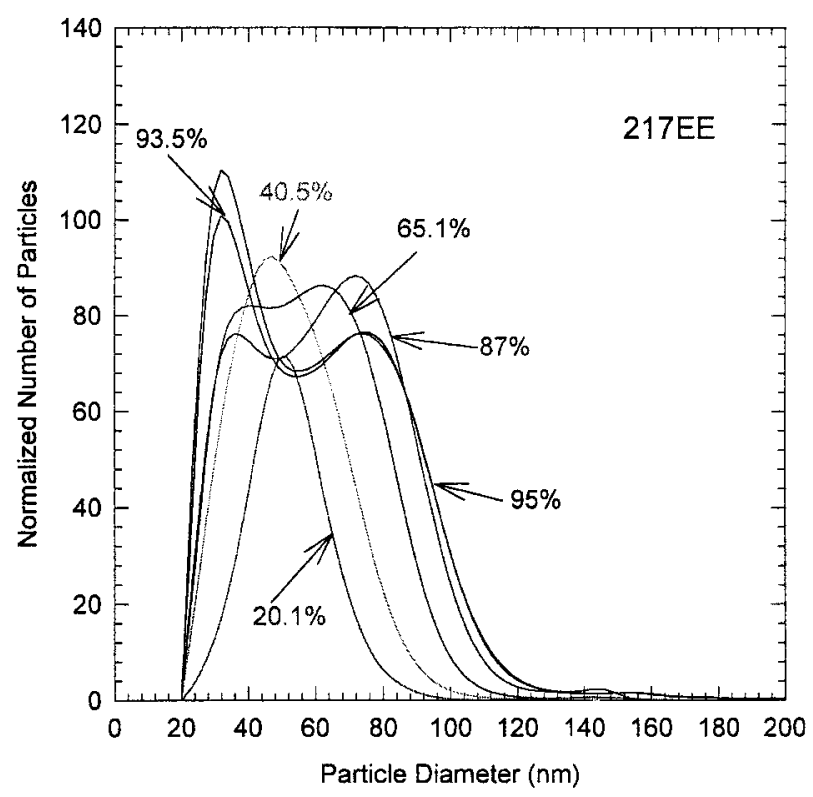

(c)

Figure 8. Comparisons of the normalized PSDs obtained by CHDF as a function of conversion for (a) Poval 217 (low degree of blockiness), (b) Poval 217E (medium degree of blockiness), and (c) Poval 217EE (highest degree of blockiness) PVAs for the batch emulsion polymerization of VAc $\left(\mathrm{K}_{2} \mathrm{~S}_{2} \mathrm{O}_{8}=0.77 \mathrm{mM}, T_{\mathrm{r}}=60{ }^{\circ} \mathrm{C}, 10 \%\right.$ solids $)$.

denced by the population of small particles persisting until the end of the reaction. Limited aggregation or flocculation also occurs among these newly generated particles and the existing polymer particles. This can be seen particularly after about $40 \%$ conversion by the bimodal distribu- tion. The observed average particle diameter is well above the size that could be achieved simply by propagation in the particles existing at the lower conversions (unimodal PSDs). For the latex prepared with the highest degree of blockiness, Poval 217EE [Fig. 8(c)], the evolution of the PSD 


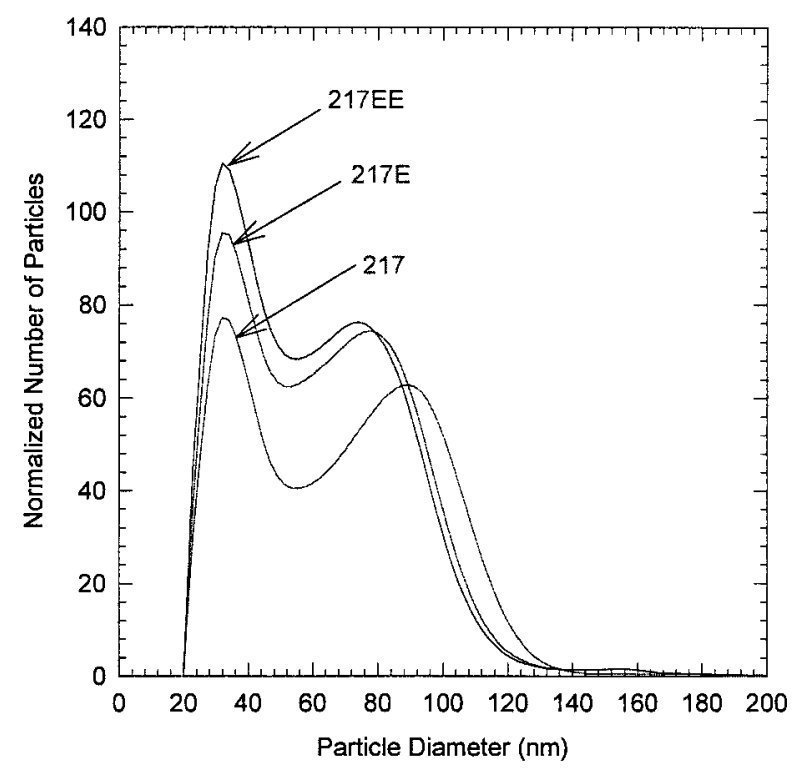

Figure 9. Comparison of the normalized PSDs obtained by CHDF for all PVAs at $95 \%$ conversion for the batch emulsion polymerization of VAc $\left(\mathrm{K}_{2} \mathrm{~S}_{2} \mathrm{O}_{8}=0.77\right.$ $\mathrm{mM}, T_{\mathrm{r}}=60{ }^{\circ} \mathrm{C}, 10 \%$ solids).

is significantly different from the latex prepared with Poval 217 [Fig. 8(a)]. The breadth of the PSD is narrower for Poval 217EE than for Poval 217. There is also a larger fraction (number) of small particles that persist until the end of the reaction for Poval 217EE, as shown in Figure 9. This is further evidence suggesting that Poval 217EE possesses better emulsifier properties and, as such, is better able to stabilize more new particles, preventing limited aggregation to a greater extent. This aggregation phenomenon is more obvious for the PVA with the lowest degree of blockiness (217) and may be due to the PVA adsorption or grafting rate being lower than the rate at which particle surface area is created. It might also be explained by differences in the distribu- tion of hydrophobic anchoring blocks and hydrophilic stabilizing loops in Poval 217 PVA compared with Poval 217EE. This is further supporting evidence that Poval 217EE is a better emulsifier than Poval 217.

Table III shows the particle size statistics of the latexes prepared with the three different PVAs both at the rate maximum and at the end of each reaction. The polydispersity index (PDI $\left.=D_{\mathrm{w}} / D_{\mathrm{n}}\right)$ at the rate maximum is lower than the PDI of the final latex for all three experiments. This reflects the continuous nucleation and aggregation phenomena occurring during the polymerizations.

For a system in which particles grow by propagation alone and nucleation ceases at the rate maximum, the PDI should decrease with conversion in the later stages. However, the fact that the PDI at the end of the polymerization is greater than the PDI at $R_{\mathrm{p} \text { max }}$ gives further evidence for the presence of limited aggregation as an important growth mechanism. The final PDIs for the latexes with the PVAs with the higher degrees of blockiness (Poval 217EE and 217E) are higher than that for the low degree of blockiness PVA (Poval 217). With a higher degree of blockiness, the capacity to stabilize a large number of small particles is increased, which generates a larger PDI.

\section{Quantitative Separations of PVAc Homopolymer and Linear and Grafted PVA}

\section{PVAc Homopolymer and Grafted PVAc Species}

The AcN/hot-water selective solubilization technique was applied to all of the samples taken at different conversions for the latexes made with Poval 217 and Poval 217EE, the PVAs with low and high degrees of blockiness, respectively. The

Table III. Particle Size Averages as Measured by CHDF for the Polymerization of VAc with PVAs with Different Degrees of Blockiness: Poval 217 (Low), Poval 217E (Medium), and Poval 217EE (High) ${ }^{\mathrm{a}}$

\begin{tabular}{llcccc}
\hline Poval PVA & Sampling Time & $D_{\mathrm{n}}(\mathrm{nm})$ & $D_{\mathrm{v}}(\mathrm{nm})$ & $D_{\mathrm{w}}(\mathrm{nm})$ & PDI \\
\hline \multirow{2}{*}{217} & $R_{\mathrm{p} \max }$ & 64.0 & 66.4 & 72.1 & 1.13 \\
& Final & 79.4 & 86.9 & 106 & 1.34 \\
$217 \mathrm{E}$ & $\sim R_{\mathrm{p} \text { max }}$ & 47.2 & 50.9 & 58.6 & 1.24 \\
& Final & 64.9 & 75.2 & 96.3 & 1.48 \\
\multirow{2}{*}{$217 \mathrm{EE}$} & $\sim R_{\mathrm{p} \max }$ & 58.3 & 59.3 & 61.8 & 1.06 \\
& Final & 77.6 & 87.0 & 136.4 & 1.76 \\
\hline
\end{tabular}

${ }^{\mathrm{a}} \mathrm{K}_{2} \mathrm{~S}_{2} \mathrm{O}_{8}=0.77 \mathrm{mM}, T_{\mathrm{r}}=60{ }^{\circ} \mathrm{C}, 10 \%$ solids. 
Table IV. Comparison of the Final Degree of Grafting during the Emulsion Polymerization of VAc with PVAs with Different Degrees of Blockiness ${ }^{a}$

\begin{tabular}{|c|c|c|c|c|c|c|c|}
\hline PVA & $\begin{array}{c}\text { PVA } \\
\text { Homopolymer } \\
\text { (g) }\end{array}$ & $\begin{array}{l}\text { Grafted } \\
\text { PVA (g) }\end{array}$ & $\begin{array}{c}\text { Grafted } \\
\text { PVAc (\%), } \\
\pm 5 \%\end{array}$ & $\begin{array}{c}\text { Linear } \\
\text { PVA (g) }\end{array}$ & $\begin{array}{l}\text { Grafted } \\
\text { PVA (g) }\end{array}$ & $\begin{array}{l}\text { Degree of } \\
\text { Grafting of } \\
\text { PVA, } \pm 5 \%\end{array}$ & $\begin{array}{c}\text { Degree of } \\
\text { Grafting of } \\
\text { PVAc, } \pm 5 \%\end{array}$ \\
\hline 217 & 4.36 & 2.53 & 36 & 2.07 & 0.87 & 29.6 & 37 \\
\hline $217 \mathrm{EE}$ & 4.17 & 2.71 & 39 & 2.65 & 0.28 & 9.6 & 39 \\
\hline
\end{tabular}

${ }^{\mathrm{a}} \mathrm{K}_{2} \mathrm{~S}_{2} \mathrm{O}_{8}=0.77 \mathrm{mM}, T_{\mathrm{r}}=60{ }^{\circ} \mathrm{C}, 10 \%$ solids, based on $100 \mathrm{~g}$ of latex.

quantitative results are shown in Table IV and Figure 10 for the PVAc homopolymer and grafted components (insoluble fractions). We obtained these by carrying out the mass balance after the separation process with eqs 2,5 , and 6 . From Figure 10, it can be observed that the final total amounts of grafted PVAc (insoluble fraction) for Poval 217EE and 217 were similar, $39 \%$ and $37 \%$ PVAc grafted, respectively. These values are within experimental error $( \pm 10 \%)$ of each other. The profiles for the grafted PVAc-conversion curves shown are similar. Initially, all the VAc that has reacted is grafted ( $\leq 2 \%$ conversion). In addition, significant amounts of additional grafting occur primarily after the disappearance of monomer droplets $(\sim 26-28 \%$ conversion). Interestingly, up to about $44 \%$ conversion, the amount of grafted PVAc is significantly higher for Poval 217 than for Poval 217EE.

It is important to emphasize that significant amounts of the AcN-insoluble fraction (containing grafted PVAc) were only found at higher conversions $(>50 \%)$, although nucleation persisted in this region. That is, significant amounts of the insoluble fraction were obtained (PVA-g-PVAc, PVAc- $b$-PVAc, and/or crosslinked PVAc) after most of the particles were formed.

We can present these results in another way to emphasize similarities and differences by graphing the ratio of PVAc grafted to VAc reacted (the fraction of PVAc that is AcN-insoluble), as shown in Figure 11. Both sets of data begin the same by starting with complete grafting of the PVAc (presumably to the PVA), as indicated previously. This fraction decreases rapidly with conversion for both systems, although this decrease is significantly larger for the polymerization employing Poval 217EE. Both systems show a minimum; this occurs at about $28 \%$ conversion for $217 \mathrm{EE}$, whereas it appears at about $50 \%$ for 217 . The two sets of data converge at about $55 \%$ conversion, with the fraction of $\mathrm{AcN}$-insoluble material in- creasing linearly to high conversions. The final amounts constitute about $40 \%$ of the PVAc formed in the reactions.

It is known from the literature that in the emulsion (and bulk) polymerization of VAc, significant branching and crosslinking occurs after about $40 \%$ conversion. ${ }^{20}$ As such, it is speculated that the insoluble fraction obtained during the polymerization can be primarily attributed to PVAc grafted onto PVA before about 50\% conversion and primarily branched and crosslinked PVAc after 50\% conversion, as depicted in Figure 11. On the basis of these results, it is speculated that the grafting of PVAc at low conversions is dependent on the properties of PVA; that is, the more PVA acts as an emulsifier (e.g., adsorbing at the oil/water interface), the less VAc is grafted. The stronger adsorption tendency and the pseudomicellar conformation of Poval 217EE PVA chains in the aqueous phase enhance the particle nucleation, minimizing the amount of grafting taking place in solution.

\section{Water-Soluble (Linear) PVA and Grafted PVA}

After the AcN selective solubilization process was completed for each latex sample, the hot-water selective solubilization process was performed on the AcN-insoluble material. The amount of linear PVA was calculated from the water-soluble fraction, and the amount of grafted PVA was calculated with eqs 7 and 8. The amount of PVA grafted (g per $100 \mathrm{~g}$ of latex) versus conversion is displayed in Figure 12 $(a, b)$ for the emulsion polymerizations of VAc with Poval 217 and Poval $217 \mathrm{EE}$, respectively. The amount of water-soluble PVA and the amount of PVA in the recipe are also displayed in these figures for comparison. Figure 12(a) shows that for Poval 217, the amount of grafted PVA only increased slightly after 15\% conversion until the end of the polymerization, where about $29 \%$ of the PVA in the recipe was 


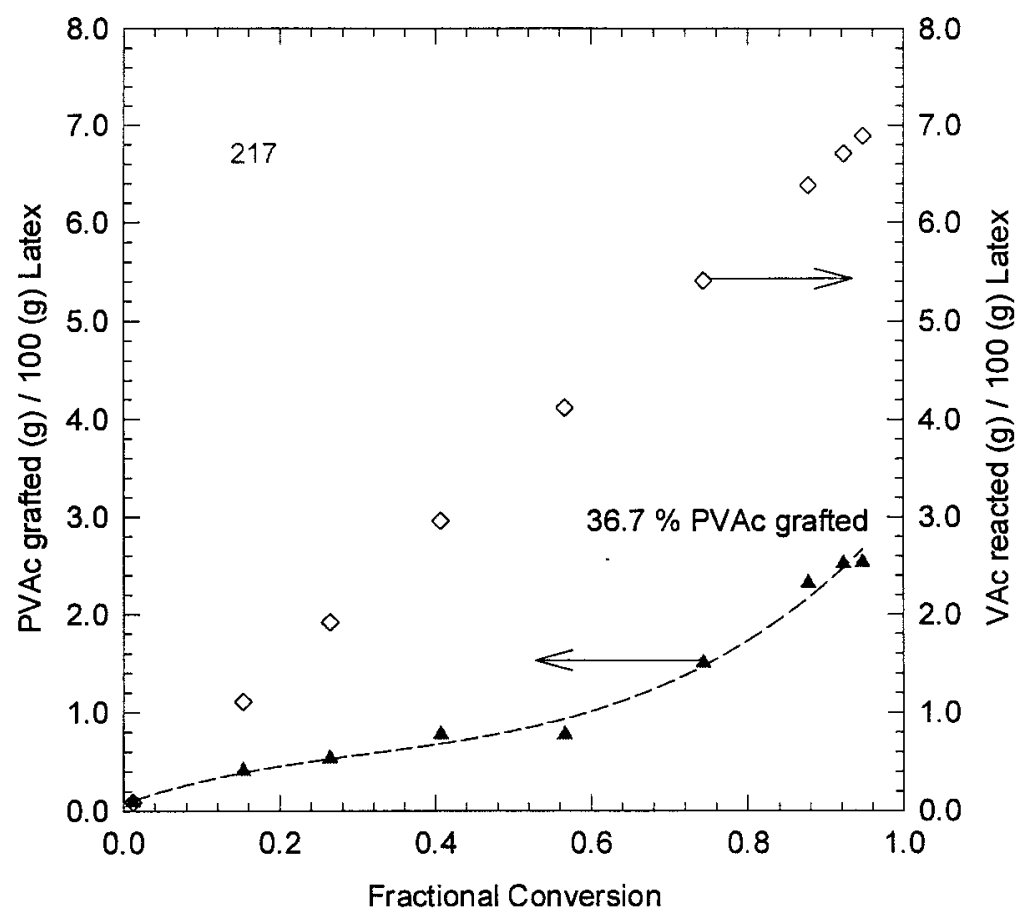

(a)

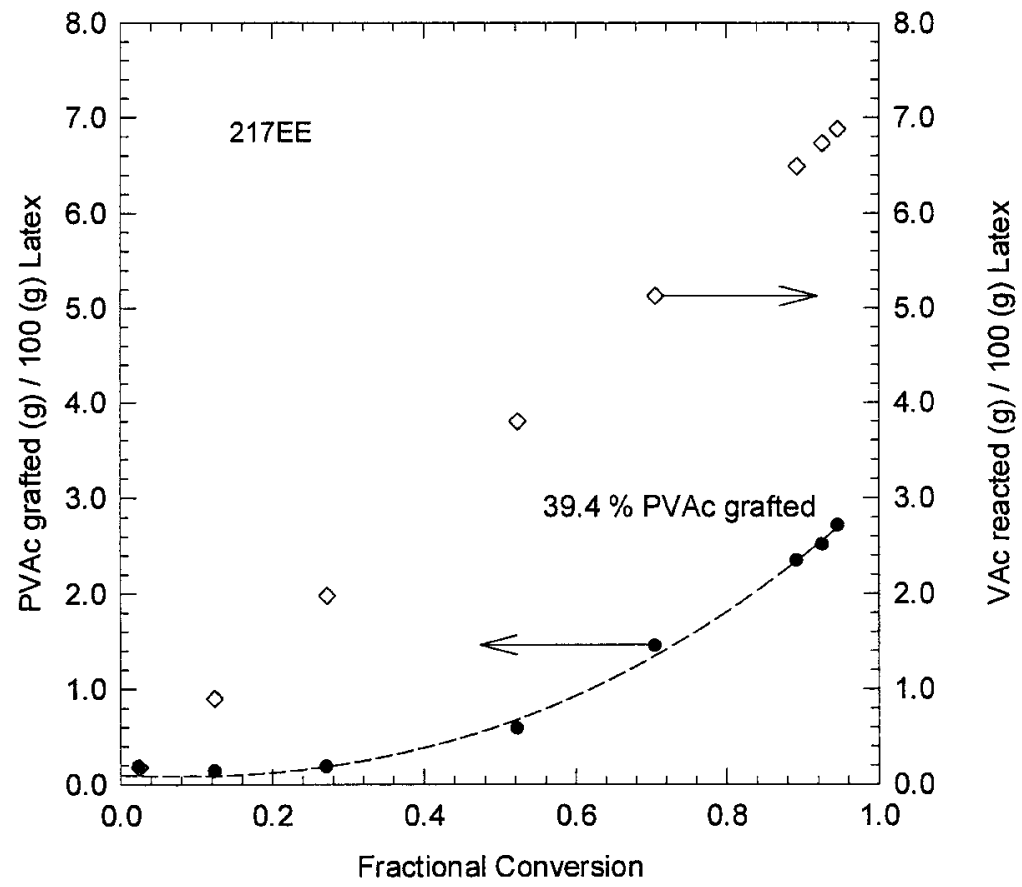

(b)

Figure 10. PVAc grafted (g/100 g of latex) and VAc reacted (g/100 g of latex) versus conversion for the emulsion polymerization of VAc with (a) Poval 217 (low) and (b) Poval 217EE (high) PVAs $\left(\mathrm{K}_{2} \mathrm{~S}_{2} \mathrm{O}_{8}=0.77 \mathrm{mM}, T_{\mathrm{r}}=60{ }^{\circ} \mathrm{C}, 10 \%\right.$ solids $)$.

grafted. Apparently, most of the grafting occurred in the initial $15 \%$ conversion, where most of the particle nucleation occurred. However, for Poval
217EE, only $10 \%$ of the PVA was grafted by the end of the polymerization, as shown in Figure 12(b). The profile of grafting for Poval 217 in- 


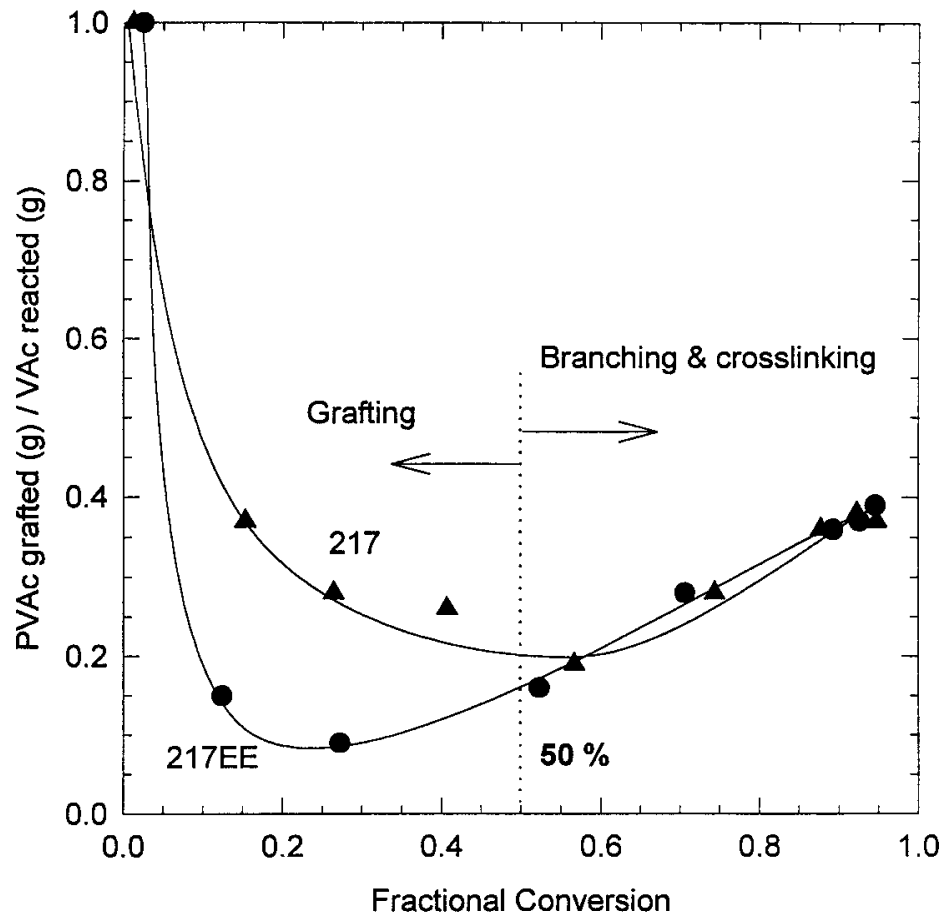

Figure 11. PVAc grafted ( $\mathrm{g}$ )/VAc reacted ( $\mathrm{g} / 100 \mathrm{~g}$ of latex) versus conversion for the emulsion polymerization of VAc with Poval 217 (low) and 217EE (high) PVAs $\left(\mathrm{K}_{2} \mathrm{~S}_{2} \mathrm{O}_{8}\right.$ $=0.77 \mathrm{mM}, T_{\mathrm{r}}=60{ }^{\circ} \mathrm{C}, 10 \%$ solids $)$.

creased beyond $R_{\mathrm{p} \text { max }}(\sim 28 \%$ conversion) and thus beyond the disappearance of monomer droplets.

This can be more comprehensively illustrated when the amount of PVA grafted relative to the total amount in the recipe (fraction grafted) is plotted as a function of conversion for both Poval 217 and Poval 217EE, as shown in Figure 13. The amount of grafted PVA is clearly dependent on the grade of PVA used: the higher the degree of blockiness of the PVA, the lower the amount of grafted PVA.

\section{Degree of Grafting of PVA}

The degree of grafting of PVA is defined as the amount of PVAc grafted (AcN-insoluble) relative to the amount of PVA grafted $(\mathrm{g} / \mathrm{g})$. This is plotted in Figure 14 as a function of conversion. This quantity is considered to be a measure of the average degree of grafting $\left(\mathrm{DG}_{\mathrm{PVA}}\right)$, which is the summation of the number of PVAc chains $\left(n_{i}\right)$ of various chain lengths $\left(\mathrm{DP}_{i}\right)$ grafted to the PVA chains $\left(n_{\mathrm{PVA}}\right)$, as given by eq 20 :

$$
\mathrm{DG}_{\mathrm{PVA}}=\frac{\mathrm{MW}_{\mathrm{VAc}} \sum_{i} n_{i} \mathrm{DP}_{i}}{n_{\mathrm{PVA}} \overline{\mathrm{MW}}_{\mathrm{PVA}}}
$$

However, although the total insoluble fraction after AcN and hot-water extractions contain both grafted PVA chains and branched/crosslinked PVAc, it is important to note that the amount of grafted PVA is a measure of the amount of waterinsoluble material, as calculated from eqs 2 and 4 . That is, a certain amount of PVAc has to graft onto the PVA chain to render it hydrophobic enough to precipitate as the water-insoluble fraction during the hot-water selective solubilization step. Vanderhoff ${ }^{21}$ predicted the limiting DP of pure PVAc oligomers in an aqueous solution to be about 1320 . However, when Hergeth et al. ${ }^{22}$ polymerized VAc in an aqueous solution without any emulsifier present and at a monomer concentration far below its saturation concentration in water, they found an average DP of about 9-11 (molecular weight $=950$ ), attributing the discrepancy to transfer reactions in the aqueous phase that Vanderhoff did not consider in his calculations. Magallanes González et al. ${ }^{10}$ found from gel permeation chromatography measurements that the molecular weight limit of the water solubility of PVAc oligomers could be placed in the range of $2600-3000 \mathrm{~g} / \mathrm{mol}$, which represents a DP of 2935. Earhart et al. ${ }^{23}$ found that the degree of hydrolysis of the grafted PVA had to decrease from 88 to $82 \%$ hydrolyzed before the grafted chain 


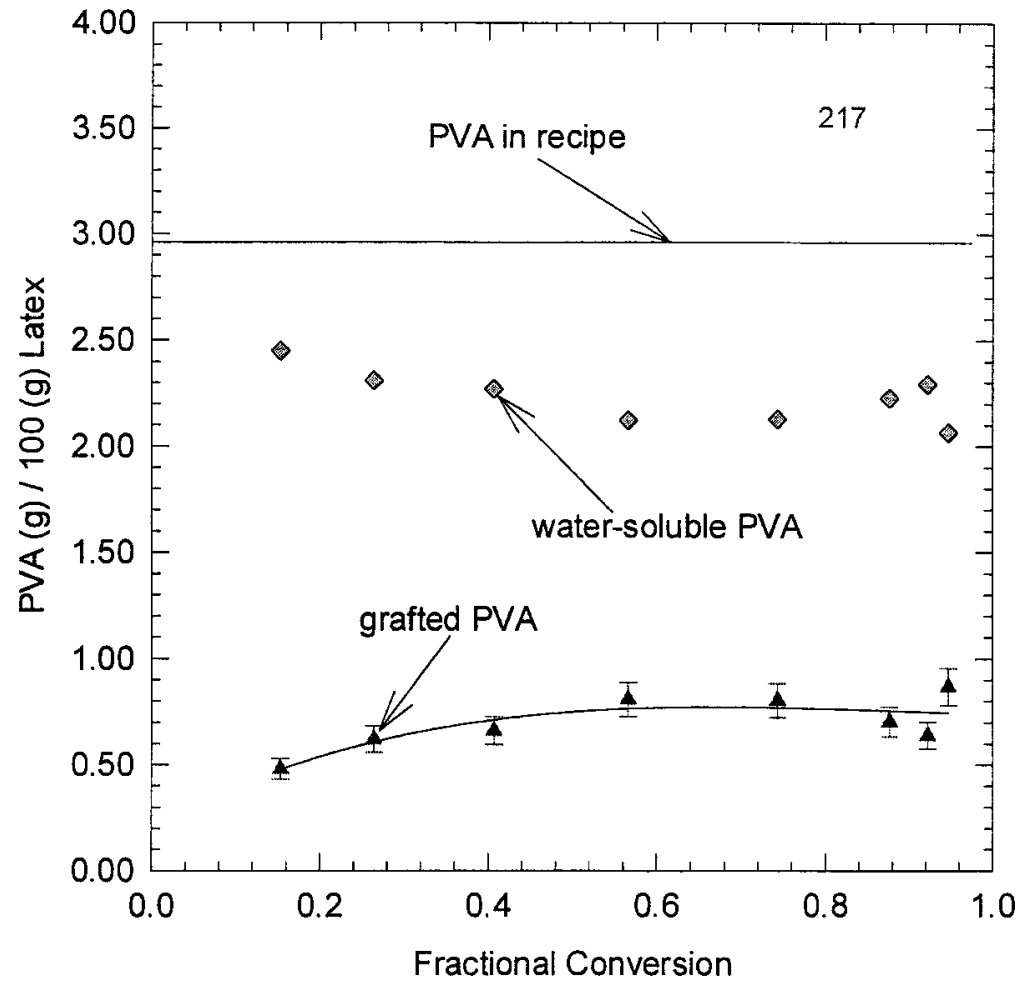

(a)

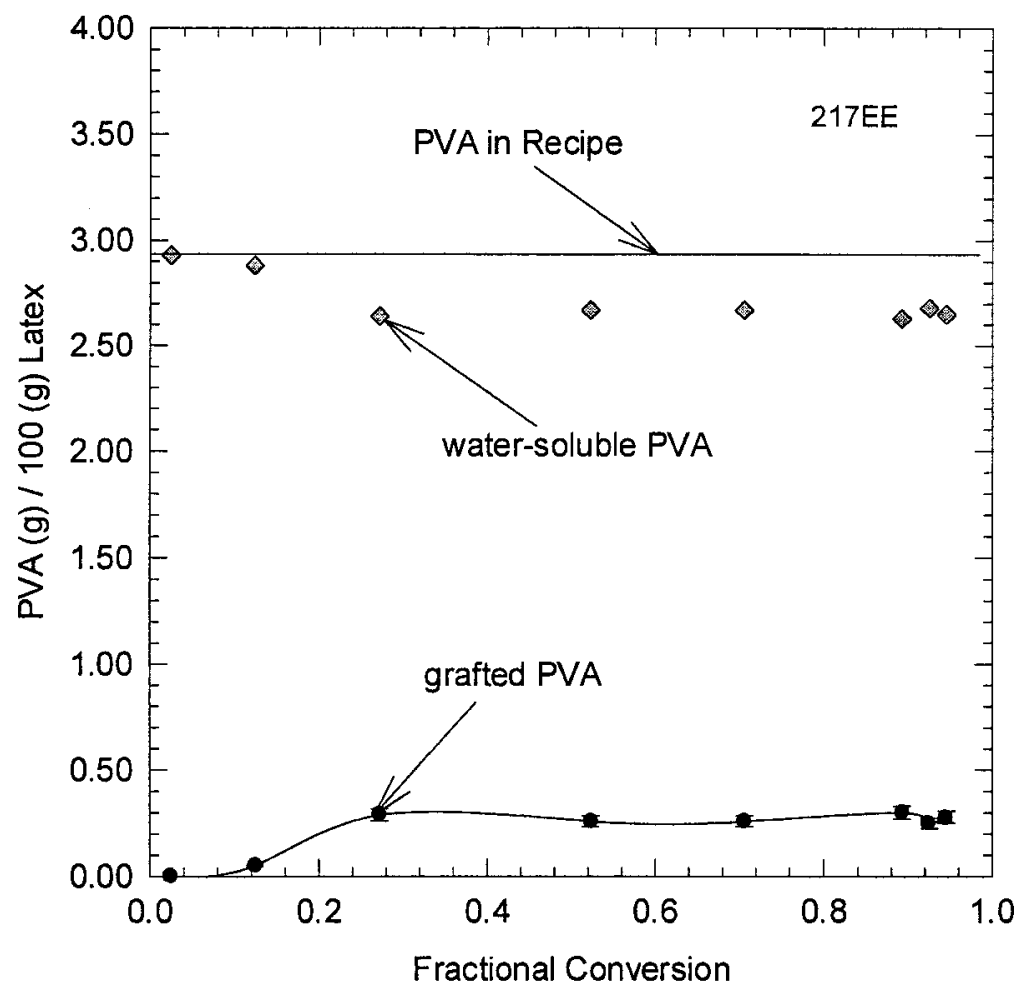

(b)

Figure 12. Grafted and ungrafted PVA ( $\mathrm{g} / 100 \mathrm{~g}$ of latex) versus conversion for the emulsion polymerization of VAc with (a) Poval 217 (low) and (b) Poval 217EE (high) PVAs $\left(\mathrm{K}_{2} \mathrm{~S}_{2} \mathrm{O}_{8}=0.77 \mathrm{mM}, T_{\mathrm{r}}=60{ }^{\circ} \mathrm{C}, 10 \%\right.$ solids $)$. 


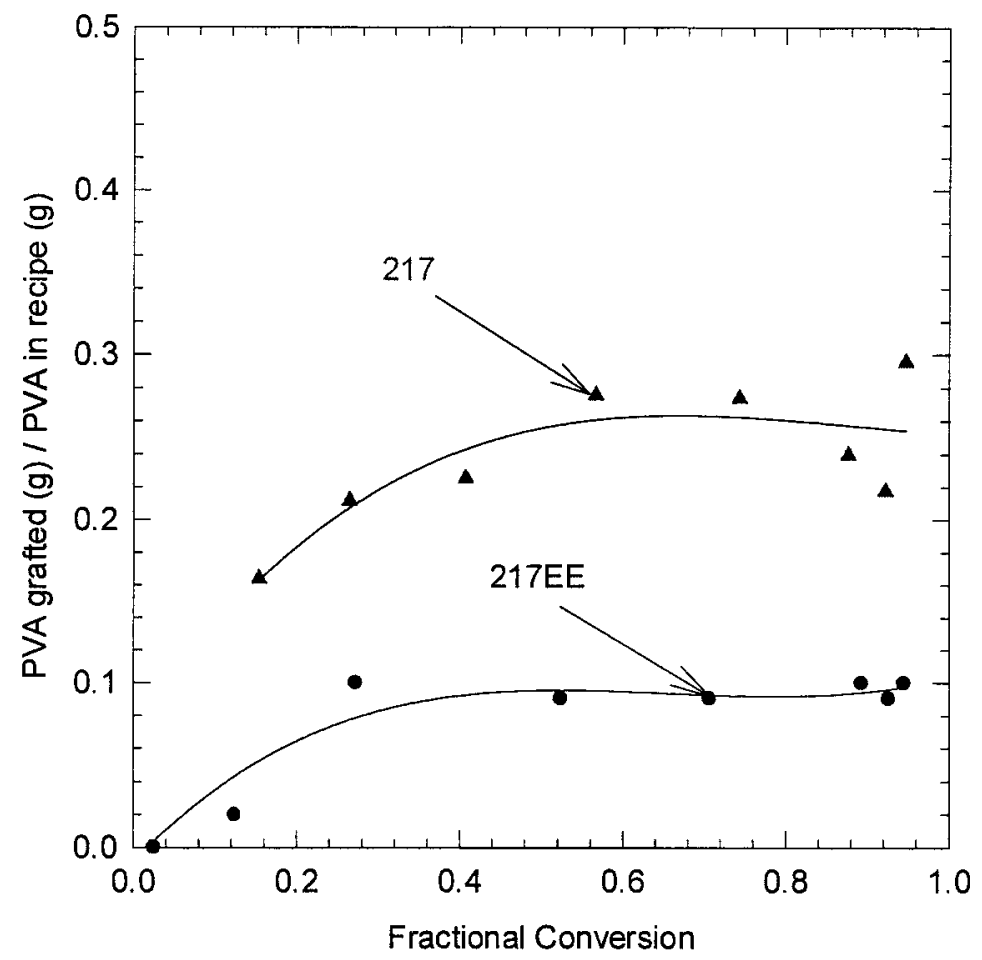

Figure 13. PVA grafted (g)/PVA in recipe (g/100 $\mathrm{g}$ of latex) versus conversion for the emulsion polymerization of VAc with Poval 217 (low) and 217EE (high) PVAs $\left(\mathrm{K}_{2} \mathrm{~S}_{2} \mathrm{O}_{8}\right.$ $=0.77 \mathrm{mM}, T_{\mathrm{r}}=60^{\circ} \mathrm{C}, 10 \%$ solids $)$.

would precipitate. Because of the extended conformation of Poval 217 in the aqueous phase (experimental evidence of the aqueous phase conformation of Poval 217 has been presented elsewhere $^{9}$ ), more grafting took place on these chains. For Poval 217EE, there is less PVA grafted but also less PVAc grafted than for Poval 217.

From Figure 14, it is observed that there is more PVAc grafted per chain of PVA for Poval 217EE. In each polymerization, the same number of chains of Poval 217 and 217EE are present, and they possess similar molecular weights. As such, it can be speculated from Figure 14 that, for Poval $217 \mathrm{EE}$, there is a greater amount of PVAc grafted onto each PVA chain. The insoluble PVAc could also result from branching/crosslinking inside the particles, but the amount of PVA grafted is less because everything may be adsorbed at the particle surface; therefore, the ratio could appear higher.

The PVA with the higher degree of blockiness (Poval 217EE) can be adsorbed faster and more strongly at the surface of the nucleated particles and thus will reside a shorter time in the aqueous phase, where the grafting reactions can occur. Therefore, there is less PVA and PVAc grafted in the early stages of the polymerization up to about $40 \%$ conversion. In contrast, Poval 217, being more weakly adsorbed at the particle surface, has a higher concentration in the aqueous phase and could be more easily grafted, increasing both the grafted amounts of PVA and PVAc in the initial stages of the polymerization. These facts suggest that the grafting reactions of PVA in the polymerization of VAc initiated by KPS (water-soluble initiator) take place primarily in the aqueous phase and are less pronounced at the particle surface.

As such, the grafting of PVA is primarily a solution event, as illustrated by both experiments. Consequently, as a result of the Poval 217 PVA being more extended in solution, grafting continues to occur for an extended period even beyond the disappearance of monomer droplets. However, for Poval 217EE, it appears that once the PVA is bound to the surface of the particles (either grafted or adsorbed), no more grafting of the PVA occurs.

The difference between the amount of PVA grafted and the amount of insoluble PVAc (grafted or crosslinked) can also be explained by the higher reactivity of the VAc radical (monomeric 


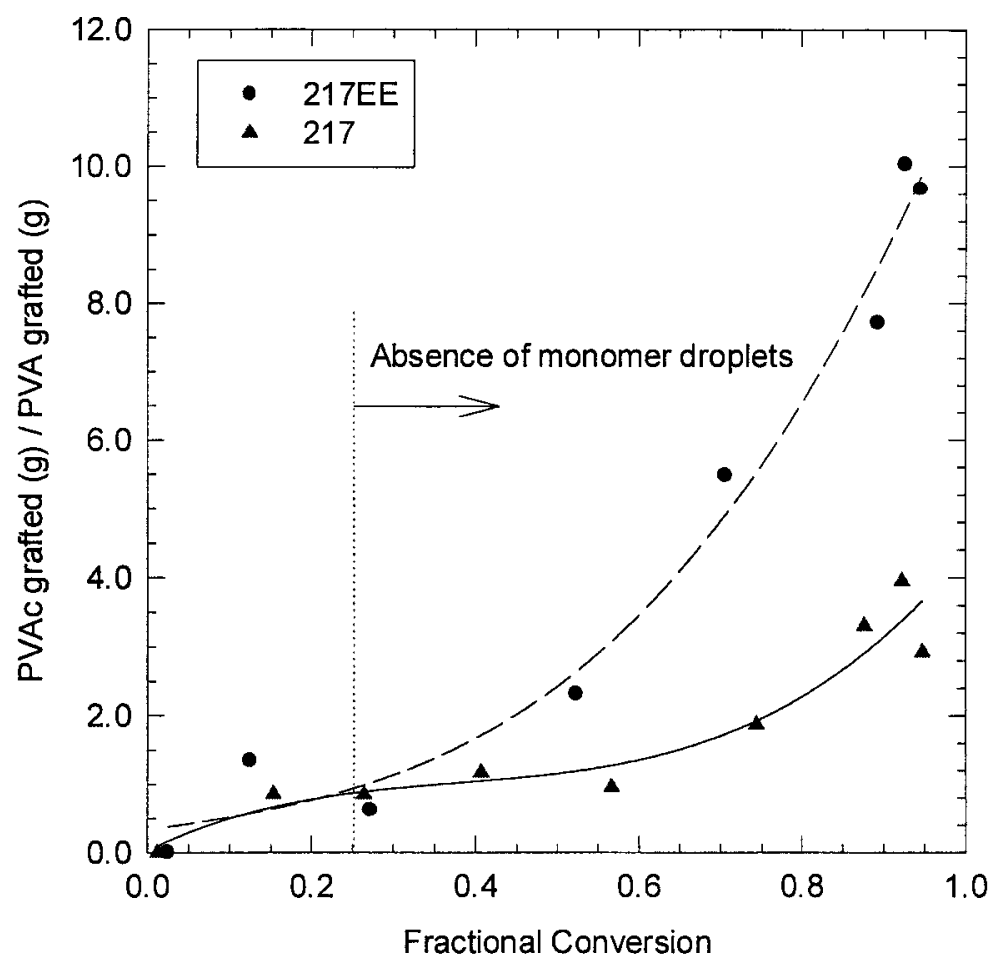

Figure 14. PVAc grafted (g)/PVA grafted (g/100 g of latex) versus conversion for the emulsion polymerization of VAc with Poval 217 (low) and 217EE (high) PVAs $\left(\mathrm{K}_{2} \mathrm{~S}_{2} \mathrm{O}_{8}\right.$ $=0.77 \mathrm{mM}, T_{\mathrm{r}}=60^{\circ} \mathrm{C}, 10 \%$ solids $)$.

radical) with VAc monomer or PVAc compared with PVA. Because grafting is primarily an aqueous phase event and these grafting reactions take place in the aqueous phase, this difference is emphasized more so for Poval 217EE than for Poval 217 because of the lack of steric hindrance (to the grafting sites $\mathrm{CH}$ and $\mathrm{CH}_{3}$ ) provided by the extended conformation of the less blocky PVA in the aqueous phase, which enhances the VAc grafting onto PVA chains. This is also observed qualitatively by the texture of the AcN-insoluble (AcN/ Ins) fraction obtained during the selective solubilization experiments. For the final samples, the AcN/Ins fraction of Poval 217EE is less swollen in AcN than that of Poval 217 because Poval 217EE contains a lower amount of PVAc grafted onto the PVA and, as such, is less readily swollen by AcN.

\section{Number of Grafted PVA Chains per Particle}

In an attempt to further determine the role of grafting during particle nucleation, we made a plot of the number of grafted PVA chains per particle as a function of conversion (Fig. 15). The following questions arise: Does grafting directly lead to the nucleation of polymer particles [by collapse of the grafted chain (PVA-g-PVAc) when it exceeds its critical chain length]? Or does polymerization of VAc in the aqueous phase lead to the formation of particles (homogeneous nucleation) followed by adsorption of PVA to stabilize the particles that are already formed by subsequently becoming grafted at the surface of the particle?

Figure 15 illustrates that for Poval 217, the number of grafted PVA chains per particle decreased as a function of conversion. This is in contrast to Poval 217EE, for which the number of grafted PVA chains per particle was much lower and remained relatively constant during the polymerization. For Poval 217EE, its blockier character may cause it to adsorb more strongly on the particle surface and provide stabilization without the extensive need for grafting. However, for Poval 217, its less blocky character may require more extensive grafting to enhance its adsorption characteristics. Its more extended conformation in solution also lends itself to more extensive grafting early in the reaction.

The data in Figure 15 seem to indicate that the grafting does not have an overriding effect on particle nucleation. In the initial stages, grafting 


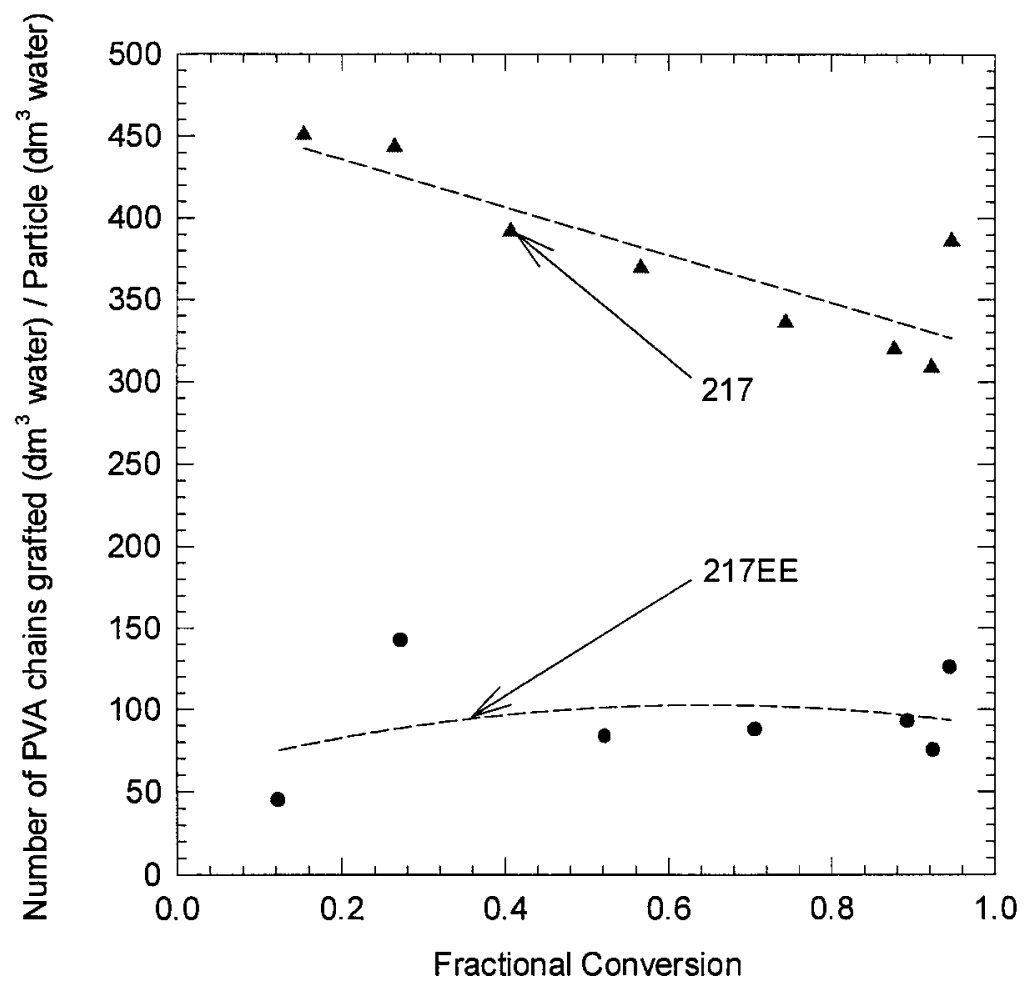

Figure 15. Number of grafted PVA chains per particle versus conversion for the emulsion polymerization of VAc with Poval 217 (low) and 217EE (high) PVAs $\left(\mathrm{K}_{2} \mathrm{~S}_{2} \mathrm{O}_{8}\right.$ $=0.77 \mathrm{mM}, T_{\mathrm{r}}=60^{\circ} \mathrm{C}, 10 \%$ solids $)$.

takes place as a chain-transfer event with a high concentration of PVA chains in the aqueous phase. When particle nucleation reaches such an extent that most of the PVA is adsorbed at the particle surface, grafting of PVA stops or decreases strongly. For Poval 217, where homogeneous nucleation continues to higher conversions (because of weaker PVA adsorption and the ease of redistribution between the particles), the number of grafted PVA chains per particle has a continuous decline.

\section{Surface Area Occupied by Grafted PVA Chains}

Ahmed $^{24}$ measured the adsorption density of $88 \%$ partially hydrolyzed PVA (Airvol 523; medium molecular weight $\sim 80,000 \mathrm{~g} / \mathrm{mol}$; Air Products \& Chemicals, Inc.) on polystyrene latex particles via the adsorption isotherm technique. On adsorption, partially hydrolyzed PVA shows specific interactions with the substrate: the more hydrophobic acetyl groups adsorb preferentially on the hydrophobic polystyrene surfaces. The adsorption density at the apparent plateau (surface saturation) for Airvol 523 was found to be $9.86 \mathrm{mg} / \mathrm{m}^{2}$. Thus, the area occupied by one molecule of Airvol
523 PVA (assuming an average molecular weight of $80,000 \mathrm{~g} / \mathrm{mol}$ ) was about $1330 \AA^{2}$. In the emulsion polymerization of VAc in the presence of PVAs with different degrees of blockiness, if it is assumed that all of the grafted PVA chains are on the surface and only grafted chains are on the surface, then the area occupied by a PVA chain $\left(a_{\mathrm{s}}\right)$ can be estimated and compared with the experimental value obtained by Ahmed.

Table V shows the results for the area occupied by one grafted PVA chain as a function of the degree of blockiness of PVA, calculated with the surface-average diameter obtained from $\mathrm{CHDF}$ particle size measurements. The area occupied by one chain $\left(a_{\mathrm{s}}\right)$ is large compared with the saturation limit obtained by Ahmed for the adsorption of PVA onto polystyrene latexes. It can be postulated from these results that there is a large amount of available space on the surface of the PVAc particles for the adsorption of ungrafted PVA.

\section{SUMMARY AND CONCLUSIONS}

The emulsion polymerization of VAc in the presence of PVAs with differing degrees of blockiness 
Table V. Area Occupied per Molecule of Grafted PVA for the Final Latex Particles Formed during the Emulsion Polymerization of VAc with PVAs with Different Degrees of Blockiness ${ }^{a}$

\begin{tabular}{|c|c|c|}
\hline $\begin{array}{l}\text { Poval } \\
\text { PVA }\end{array}$ & $\begin{array}{l}\text { No. of Grafted PVA } \\
\text { Chains per Particle }\end{array}$ & $\begin{array}{l}\text { Area Occupied } \\
\text { by One Grafted } \\
\text { PVA Chain }\left(\AA^{2}\right)\end{array}$ \\
\hline 217 & 385 & 3,362 \\
\hline $217 \mathrm{EE}$ & 125 & 24,870 \\
\hline
\end{tabular}

${ }^{\mathrm{a}} \mathrm{K}_{2} \mathrm{~S}_{2} \mathrm{O}_{8}=0.77 \mathrm{mM}, T_{\mathrm{r}}=60{ }^{\circ} \mathrm{C}, 10 \%$ solids.

was investigated. The reaction kinetics, particle number evolution, and development of grafting were followed. No discernable relationship between $N_{\mathrm{p}}$ and $R_{\mathrm{p}}$ was observed. Rates of polymerization were found to be relatively independent of the PVA type. An analysis of the reaction kinetics showed low values of $\bar{n}$, characteristic of SmithEwart case 1 kinetics. No constant rate interval II was noted, and interval III was characterized by the presence of the gel effect, seen as a shoulder in $R_{\mathrm{p}}$ curves.

$N_{\mathrm{p}}$ was found to be dependent on the PVA blockiness. The PVA with the higher degree of blockiness led to the nucleation and stabilization of more particles. Nucleation continued to high conversions for the more random PVA (Poval 217), whereas it appeared to stop at intermediate conversions for the blockier stabilizer (Poval 217EE). All systems exhibited limited aggregation of the polymer particles during the polymerization process, differing in the extent and evolution.

At higher conversions $(>55 \%)$, the total amount of grafted PVAc was independent of the degree of blockiness of the PVA, as evidenced by similar grafting efficiencies of PVAc for both PVAs. The amount of grafted PVAc was 2.53 $\mathrm{g} / 100 \mathrm{~g}$ of latex for Poval 217, representing $37 \%$ of the reacted VAc, and $2.71 \mathrm{~g} / 100 \mathrm{~g}$ of latex for Poval 217EE, representing $39 \%$ of the reacted VAc. The amount of grafted PVA decreased with the increase in the degree of blockiness of the PVA, that is, $0.87 \mathrm{~g} / 100 \mathrm{~g}$ of latex for Poval 217, representing a grafting efficiency of about $30 \%$, compared with $0.28 \mathrm{~g} / 100 \mathrm{~g}$ of latex for Poval $217 \mathrm{EE}$, representing about $10 \%$ grafting efficiency.

The greatest amount of grafting of the PVA stabilizer occurred early in the reactions $(<25 \%$ conversion), presumably contributing primarily to the stabilization of the particles. Further nu- cleation without measurable grafting indicates a homogeneous nucleation mechanism with adsorption of aqueous phase PVA and redistribution of some previously adsorbed PVA, thus providing stability.

Financial support from the Emulsion Polymers Liaison Program is greatly appreciated.

\section{REFERENCES AND NOTES}

1. Napper, D. H. Polymeric Stabilization of Colloidal Dispersions; Academic: San Diego, 1983.

2. Pramojaney, N. Ph.D. Dissertation, Lehigh University, 1982.

3. Finch, C. A. Polyvinyl Alcohol: Properties and Applications; Wiley: New York, 1973.

4. Dunn, A. S. In Emulsion Polymerization of Vinyl Acetate; El-Aasser, M. S.; Vanderhoff, J. W., Eds.; Applied Science: New York, 1981; p 11.

5. Hartley, F. D. J Polym Sci 1959, 34, 397.

6. Gilmore, C. M.; Poehlein, G. W.; Schork, F. J. J Appl Polym Sci 1993, 48, 1449.

7. Dunn, A. S. In 11th Annual Short Course Notes: Advances in the Emulsion Polymerization and Latex Technology; El-Aasser, M. S., Ed.; Emulsion Polymers Institute, Lehigh University: Bethlehem, PA, 1980; Vol. II.

8. Dimonie, V.; Donescu, D.; Munteanu, M.; Hagiopol, C.; Gavãt, I. Rev Romaine Chim 1974, 19, 903.

9. Budhlall, B. M. Ph.D. Dissertation, Lehigh University, 2000.

10. Magallanes González, G. S.; Dimonie, V. L.; Sudol, E. D.; Yue, H. J.; Klein, A.; El-Aasser, M. S. J Polym Sci Part A: Polym Chem 1996, 34, 849.

11. Azeotropic Data III; Gould, R. F., Ed.; Advances in Chemistry Series 116; American Chemical Society: Washington, DC, 1973.

12. Varela de la Rosa, L. Ph.D. Dissertation, Lehigh University, 1996.

13. Varela de la Rosa, L.; Sudol, E. D.; El-Aasser, M. S.; Klein, A. J Polym Sci Part A: Polym Chem 1996, 34, 461.

14. Friis, N.; Hamielec, A. E. In Emulsion Polymerization; Piirma, I.; Gardon, J. L., Eds.; ACS Symposium Series 24; American Chemical Society: Washington, DC, 1976; p 82.

15. Magallanes González, G. S. Ph.D. Dissertation, Lehigh University, 1996.

16. Hutchinson, R. A.; Richards, J. R.; Aronson, M. T. Macromolecules 1994, 27, 4530.

17. Chang, K. H. S.; Litt, M. H.; Nomura, M. In Emulsion Polymerization of Vinyl Acetate; El-Aasser, M. S.; Vanderhoff, J. W, Eds.; Applied Science: Englewood, NJ, 1981; p 89.

18. Smith, W. V.; Ewart, R. H. J Chem Phys 1948, 16, 592. 
19. Nomura, M.; Harada, M.; Eguchi, W.; Nagata, S. In Emulsion Polymerization; Piirma, I.; Gardon, J. L.; Eds.; ACS Symposium Series 24; American Chemical Society: Washington, DC, 1976; p 102.

20. Motoyama, T. Kunststoffe 1960, 5, 1, 33.

21. Vanderhoff, J. W. J Polym Sci Polym Symp 1985, $72,161$.

22. Hergeth, W. D.; Lebek, W.; Kakuschke, R.; Schmutzler, K. Makromol Chem 1991, 192, 265.
23. Earhart, N.; Vanderhoff, J. W.; Dimonie, V. L.; El-Aasser, M. S. Makromol Chem Macromol Symp 1990, 35/36, 477-497.

24. (a) Ahmed, S. M. Ph.D. Dissertation, Lehigh University, 1984; (b) Ahmed, M. S.; El-Aasser, M. S.; Vanderhoff, J. W. In Polymer Adsorption and Dispersion Stability; Goddard, E. D.; Vincent, B., Eds.; ACS Symposium Series 240; American Chemical Society: Washington, DC, 1984; p 77. 